COMMUNICATIONS IN

ANALYSIS AND GEOMETRY

Volume 14, Number 1, 183-214, 2006

\title{
From spatially periodic instantons to singular monopoles
}

\author{
Benoit Charbonneau ${ }^{1}$
}

\begin{abstract}
The main result is a computation of the Nahm transform of a $\mathrm{SU}(2)$-instanton over $\mathbb{R} \times T^{3}$, called spatially-periodic instanton. It is a singular monopole over $T^{3}$, a solution to the Bogomolny equation, whose rank is computed and behavior at the singular points is described.
\end{abstract}

\section{Introduction.}

Heuristically, there is a correspondence, called the Nahm transform, between

1. solutions to the anti-self-dual (ASD) equation, or its appropriate dimensional reduction, on the quotient of $\mathbb{R}^{4}$ by a closed subgroup $\Lambda$ of $\mathbb{R}^{4}$, and satisfying a finite energy condition, and

2. solutions to some associate equation satisfying some boundary condition on the quotient of $\mathbb{R}^{4^{*}}$ by the dual subgroup $\Lambda^{*}=\left\{f \in \mathbb{R}^{4^{*}}\right.$ $f(\Lambda) \subset \mathbb{Z}\}$.

This heuristic comes from a re-engineering due to Nahm [22] of the ADHM construction of instantons on $\mathbb{R}^{4}[1]$. Nahm's approach has the advantage of being transportable to quotients by non-trivial subgroup $\Lambda$ as well, with some ad hoc efforts necessary in each case.

Nahm gave an outline of the correspondence for classical instantons $(\Lambda=$ $\{0\})$ and for monopoles on $\mathbb{R}^{3}(\Lambda=\mathbb{R})$. Corrigan and Goddard in [10] completed the details of the ADHM construction following Nahm's guideline, while Hitchin in [13] completed the story for $\mathrm{SU}(2)$-monopole on $\mathbb{R}^{3}$. In [23], Nakajima rendered Hitchin's proof more parallel to the ADHM story.

This framework guided several other authors in the quest for an understanding of other moduli spaces of instantons (or their appropriate dimensional reduction) on various quotients of $\mathbb{R}^{4}$ : for instantons on $T^{4}$, see $[27,5]$;

\footnotetext{
${ }^{1}$ This research was supported by NSERC PGS A-B and MIT.
} 
for monopoles of other classical groups, see [14]; for calorons, or instantons on $S^{1} \times \mathbb{R}^{3}$, see $[25,24]$; for instantons on $T^{2} \times \mathbb{R}^{2}$, see $[19,15,16,17,4]$; and for monopoles on $\mathbb{R}^{2} \times S^{1}$, see $[8,9,7]$. Marcos Jardim wrote a survey paper [18] on the Nahm transform, and the reader is invited to consult it for some insights on an even more general framework in which to place the above referenced literature and the present paper.

Apart from some numerical approximations and remarks in [28] and a computation of the Nahm transform of charge 1 instantons in [29], the case of the spatially periodic instantons, instantons on $\mathbb{R} \times T^{3}$, has been largely ignored. The present paper starts the groundwork necessary to close that gap. We prove here that the Nahm transform of an instanton on $\mathbb{R} \times T^{3}$ is a singular monopole on $T^{3}$ with specific behavior at the singular points.

This paper is organized as follows. The main result on the Nahm transform of instantons on $\mathbb{R} \times T^{3}$ and its singular behavior is spelled out in Section 5 after the adequate language is explained. Before reaching this result, it is useful to go over a brief overview of the classical ADHM construction in Section 2, then check the bigger picture of the Nahm transform heuristic in Section 3, and then zoom in on the Fredholmness properties of the Dirac operators on $\mathbb{R} \times T^{3}$ in Section 4 . The proof of the result splits three ways: first, the rank of the transformed bundle is computed at the end of Section 5; then, a splitting of the transformed bundle around the singularities is developed in Section 6; and finally, the asymptotic of the Higgs field is proved in Section 7 .

\section{The classical ADHMN.}

The classical work of Atiyah, Drinfeld, Hitchin and Manin, termed ADHM construction, classifies all the solutions to the ASD equation on $\mathbb{R}^{4}$, up to gauge equivalence. Once viewed under the umbrella of the Nahm transform heuristic, thus adding an $\mathrm{N}$ to form $A D H M N$, the classification is as follows.

A connection $A$ on a $\mathrm{SU}(n)$-bundle $E$ over $\mathbb{R}^{4}$ whose curvature $F_{A}$ satisfies the ASD equation $* F_{A}=-F_{A}$ and the finite energy condition $\int_{\mathbb{R}^{4}}\left|F_{A}\right|^{2}<\infty$ gives rise, through an analysis of its Dirac operator $\mathfrak{D}_{A}$, to a set of algebraic data: two vector spaces

$$
\begin{gathered}
V=L^{2} \cap \operatorname{ker}\left(\mathfrak{P}_{A}^{*}\right), \text { and } \\
W=\text { bounded harmonic sections of } E \text { for } \nabla_{A},
\end{gathered}
$$


and five maps

$$
\begin{gathered}
\Phi_{1}, \ldots, \Phi_{4}: V \rightarrow W \\
\eta: V \rightarrow S^{+} \otimes W .
\end{gathered}
$$

Since the vector space is built using the augmented Dirac operator $\mathfrak{P}_{A}^{*}$ acting on sections of $S^{-} \otimes E$, the dimension of $V$ can be computed by some index theorem, and

$$
\operatorname{dim} V=\frac{1}{8 \pi^{2}} \int_{\mathbb{R}^{4}}\left|F_{A}\right|^{2}
$$

provided the cokernel $L^{2} \cap \operatorname{ker}\left(\mathscr{D}_{A}\right)$ is $\{0\}$. It is indeed so, as the Weitzenbock formula

$$
\mathfrak{P}_{A}^{*} \mathfrak{D}_{A}=\nabla_{A}^{*} \nabla_{A}+\operatorname{cl}\left(F_{A}^{+}\right)
$$

clearly establishes: for an instanton connection, the Clifford multiplication term vanishes and a $L^{2}$ solution $\phi$ to $\mathfrak{D}_{A}^{*} \phi=0$ must be parallel, hence 0 since $\mathbb{R}^{4}$ has infinite volume.

The map $\Phi_{i}=P m_{x_{i}}$ is the composite of the multiplication by the $i$ th coordinate, denoted $m_{x_{i}}$ and the $L^{2}$-projection $P$ on $\operatorname{ker}\left(\mathscr{D}_{A}^{*}\right)$, while the map $\eta$ encodes the asymptotic behavior of elements of $V$.

For an instanton $(E, A)$, the associated algebraic data $(V, W, \Phi, \eta)$ satisfy a non-degeneracy condition and the ADHM equation, the precise formulation of which is not important here. This "ADHM transform" places in one-to-one correspondence instantons modulo gauge equivalence with nondegenerate solutions to the ADHM equation modulo some symmetry group action. A complete description of this construction can be found in [11, Chapter 3], and in the author's thesis [6].

It is a fruitful idea to interpret the set of maps $\Phi=\left(\Phi_{1}, \ldots, \Phi_{4}\right)$ as a constant connection form

$$
\bar{B}=\Phi_{1} d x^{1}+\cdots \Phi_{4} d x^{4}
$$

on the trivial bundle $\underline{V}$ over $\mathbb{R}^{4}$ with fiber $V$. The curvature $F_{\bar{B}}$ of $\bar{B}$ splits as

$$
F_{\bar{B}}=(\mathrm{ASD} \text { part })+(\mathrm{SD} \text { part involving } \eta) .
$$

Morally, the idea is that the transformed connection $\bar{B}$ on $\mathbb{R}^{4^{*}}$, invariant under the action of $\Lambda^{*}=\mathbb{R}^{4^{*}}$, is almost anti-self-dual, and the self-dual part is determined by the asymptotic behavior of harmonic spinors. 


\section{The Nahm transform heuristic.}

The work of Nahm provides a framework in which to think about the classification of all the finite energy solutions to the ASD equation on a quotient $\mathbb{R}^{4} / \Lambda$. Philosophically, once we find the appropriate codomain for the Nahm transform to be described in this section, it should be an isomorphism. This idea has been shown to work in many cases, as explained in the introduction.

A connection $A$ on a $\mathrm{SU}(n)$-bundle $E$ over $\mathbb{R}^{4}$, invariant under the action of a closed subgroup $\Lambda$, and whose curvature $F_{A}$ satisfies the ASD equation

$$
* F_{A}=-F_{A}
$$

and the finite energy condition

$$
\int_{\mathbb{R}^{4} / \Lambda}\left|F_{A}\right|^{2}<\infty
$$

gives rise, this time, to a bundle $V$ with a connection $B$ over $\mathbb{R}^{4^{*}} / \Lambda^{*}$. Those objects are constructed in the following way.

For an element $z$ of $\mathbb{R}^{4^{*}}$, the space of $\mathbb{R}$-valued linear functions on $\mathbb{R}^{4}$, we define the bundle $L_{z}$ over $\mathbb{R}^{4}$ to be a trivial $\mathbb{C}$-bundle with connection

$$
\omega_{z}:=2 \pi i z=2 \pi i \sum_{j=1}^{4} z_{j} d x^{j} .
$$

For $z^{\prime} \in \Lambda^{*}$, the flat bundles $L_{z}$ and $L_{z+z^{\prime}}$ over $\mathbb{R}^{4} / \Lambda$, both invariant under the action of $\Lambda$, are isomorphic. We write $A_{z}$ for the connection $A \otimes 1+1 \otimes \omega_{z}$ on $E \otimes L_{z}=E$. For $z \in \mathbb{R}^{4^{*}}$, consider the operator

$$
\mathfrak{P}_{A_{z}}^{*}: \Gamma\left(\mathbb{R}^{4}, S^{-} \otimes E \otimes L_{z}\right) \rightarrow \Gamma\left(\mathbb{R}^{4}, S^{+} \otimes E \otimes L_{z}\right) .
$$

A section of the bundle $S^{-} \otimes E \otimes L_{z}$ is said to be in $L_{\Lambda}^{2}$ if it is invariant under the action of $\Lambda$ and if its $L^{2}$-norm over $\mathbb{R}^{4} / \Lambda$ is finite.

The first ingredient of the Nahm transform of the instanton $(E, A)$ is the family of vector spaces

$$
V_{z}:=L_{\Lambda}^{2} \cap \operatorname{ker}\left(\mathscr{D}_{A_{z}}^{*}\right) .
$$

Since the vector space $V_{z}$ is built using the augmented Dirac operator $\mathfrak{D}_{A_{z}}^{*}$ acting on sections of $S^{-} \otimes E$, the dimension of $V_{z}$ can often be computed by an appropriately chosen index theorem, and it is constant on connected components on which $\mathscr{D}_{A_{z}}$ is Fredholm provided the cokernel $L_{\Lambda}^{2} \cap \operatorname{ker}\left(\mathscr{D}_{A_{z}}\right)$ 
is $\{0\}$. For a quotient $\mathbb{R}^{4} / \Lambda$ of infinite volume, it is indeed so, as the Weitzenbock formula

$$
\mathfrak{D}_{A}^{*} \mathfrak{D}_{A}=\nabla_{A}^{*} \nabla_{A}+\operatorname{cl}\left(F_{A}^{+}\right)
$$

clearly establishes: for an instanton connection, the Clifford multiplication term vanishes and a $L^{2}$ solution $\phi$ to $\mathfrak{D}_{A}^{*} \phi=0$ must be parallel, hence 0 because of the infinite volume condition. For a quotient of finite volume, we must add an extra condition to ensure the cokernel is trivial.

It turns out in many cases that $\mathfrak{D}_{A_{z}}^{*}$ is not Fredholm for every $z$, which is a good thing. Suppose for example that $\mathfrak{D}_{A_{z}}^{*}$ was Fredholm everywhere when $\Lambda=\mathbb{Z}^{3}$. As we explore in this present paper, the object created by the Nahm transform is a monopole over $T^{3}$. But as one can show (see [26, Prop. $1]$ ), smooth monopoles over compact 3-manifolds are not very interesting.

Set $g_{z}(x):=e^{2 \pi i z(x)}$. Notice that for any section $\phi$ of $S^{-} \otimes E$, we have $\mathfrak{P}_{A_{z}}^{*}\left(g_{z} \phi\right)=g_{z} \mathfrak{P}_{A}^{*} \phi$. Then, for all $z^{\prime} \in \Lambda^{*}$, we have an isomorphism

$$
g_{z^{\prime}}: V_{z} \rightarrow V_{z+z^{\prime}}
$$

hence $V$ is a bundle over $\mathbb{R}^{4^{*}} / \Lambda^{*}$.

\subsection{First viewpoint: on $\mathbb{R}^{4^{*}}$, a curvature computation.}

In the understanding of the ADHM construction, it was beneficial to view the maps $\Phi_{i}$ as parts of a connection on the bundle $\underline{V}$ on $\mathbb{R}^{4^{*}}$, without passing to the quotient. We do similarly here and consider first the bundle $V$ on an open subset of $\mathbb{R}^{4^{*}}$ on which the Dirac operator is Fredholm.

We define a connection $B$ on $V$. Each fiber $V_{z}$ is in fact contained in the vector space $L_{\Lambda}^{2}\left(S^{-} \otimes E\right)$. We can then consider the trivial connection $d^{z}$ in the trivial bundle of fibers $L_{\Lambda}^{2}\left(S^{-} \otimes E\right)$, and its projection $P d^{z}$ to $V$.

The operator $\mathfrak{D}_{A_{z}}^{*} \mathfrak{D}_{A_{z}}$ should be invertible, and we use its inverse, the Green's operator $G_{A_{z}}=\left(\mathscr{D}_{A_{z}}^{*} \mathfrak{D}_{A_{z}}\right)^{-1}$, to define the projection $P$ by the formula

$$
P=1-\mathfrak{D}_{A_{z}} G_{A_{z}} \mathfrak{D}_{A_{z}}^{*}
$$

To parallel the ADHMN story, let us now compute the curvature $F_{B}$ of $B$. To simplify the notation, we set $\Omega:=2 \pi i \sum_{j=1}^{4} c l\left(d x^{j}\right) d z^{j}$. Then $\left[d^{z}, \mathfrak{D}_{A_{z}}\right]=\Omega$, and similarly for $\mathfrak{P}_{A_{z}}^{*}$. 
The curvature $F_{B}$ can be computed as follows:

$$
\begin{aligned}
\left\langle\left(P d^{z}\right)^{2} \phi, \psi\right\rangle & =\left\langle d^{z} P d^{z} \phi, \psi\right\rangle \\
& =\left\langle P d^{z} \phi, d^{z} \psi\right\rangle-\left\langle d^{z} \phi, d^{z} \psi\right\rangle \\
& =-\left\langle\mathfrak{D}_{A_{z}} G_{A_{z}} \mathfrak{D}_{A_{z}} d^{z} \phi, d^{z} \psi\right\rangle \\
& =\left\langle\mathscr{D}_{A_{z}} G_{A_{z}} \Omega \phi, d^{z} \psi\right\rangle .
\end{aligned}
$$

Let $\nu$ be the normal vector field to $S^{r-1}(R) \times T^{s}$. The integration by parts necessary to bring $\mathscr{D}_{A_{z}}$ on the right-hand side of the scalar product introduces a boundary term

$$
\partial \text {-term }:=\lim _{R \rightarrow \infty} \int_{S^{r-1}(R) \times T^{s}}\left\langle c l(\nu) G_{A_{z}} \Omega \phi, d^{z} \psi\right\rangle .
$$

Performing the said integration by parts, we obtain

$$
\begin{aligned}
\left\langle F_{B} \phi, \psi\right\rangle & =\left\langle G_{A_{z}} \Omega \phi, \nsupseteq_{A_{z}} d^{z} \psi\right\rangle+\partial \text {-term } \\
& =-\left\langle G_{A_{z}} \Omega \phi, \Omega \psi\right\rangle+\partial \text {-term } \\
& =\left\langle G_{A_{z}} \phi, \Omega \wedge \Omega \psi\right\rangle+\partial \text {-term. }
\end{aligned}
$$

In terms of the usual basis $\epsilon_{j}$ and $\bar{\epsilon}_{j}$ of respectively $\Lambda^{+}$and $\Lambda^{-}$, we have

$$
\Omega \wedge \Omega=-4 \pi^{2} \sum_{j=1}^{3}\left(c l\left(\epsilon_{j}\right) \epsilon_{j}+c l\left(\bar{\epsilon}_{j}\right) \bar{\epsilon}_{j}\right) .
$$

Since $\wedge^{+}$acts trivially on $S^{-} \otimes E$, the first term of the curvature is ASD.

In the case we are studying at this moment, the $\partial$-term is 0 .

\subsection{Second viewpoint: on a 3-dimensional quotient, the Bogolmolny equation.}

Let us now shift our perspective and look at $V$ and $\bar{B}$ from the viewpoint of the quotient. Suppose some $\mathbb{R}$ is in $\Lambda^{*}$, say as the axis $z_{1}$. In fact, suppose here that $\Lambda=\mathbb{Z}^{3}$, and thus that $\Lambda^{*}=\mathbb{R} \times \mathbb{Z}^{3}$. Set $g_{z}(x)=e^{2 \pi i x_{1} z_{1}}$. Then,

$$
g(\bar{B})=-2 \pi i P m_{x_{1}} d z^{1}+P\left(\frac{\partial}{\partial z_{2}} d z^{2}+\cdots+\frac{\partial}{\partial z_{4}} d z^{4}\right) .
$$

So using this gauge transformation, we render $\bar{B}$ independent of the $z_{1}$ coordinate. We define the Higgs field $\Phi$ by

$$
\Phi=-2 \pi i P m_{x_{1}},
$$


and the connection $B$ on $\mathbb{R}^{4 *} / \Lambda^{*}=T^{3}$ by

$$
B=P d^{z},
$$

where $z$ represents here the coordinates $\left(z_{2}, z_{3}, z_{4}\right)$ on $T^{3}$. As we just saw,

$$
g(\bar{B})=\Phi d z^{1}+B .
$$

Should we be able to prove that $\partial$-term $=0$, it would be so that $g(\bar{B})$ is ASD. It is in fact so, as we see in the next section, and thus $(B, \Phi)$ satisfies the dimensional reduction of the ASD equation

$$
\nabla_{B} \Phi=* F_{B}
$$

called the Bogomolny equation.

\section{Fredholmness of the Dirac operator.}

It is crucial now to understand exactly for which $z \in T^{3}$ the Dirac operator $\mathfrak{P}_{A_{z}}^{*}$ acting on $L^{2}$ sections of $S^{-} \otimes E$ over $\mathbb{R} \times T^{3}$ is Fredholm.

Let us start with a $\mathrm{SU}(2)$-instanton $(E, A)$ on $\mathbb{R} \times T^{3}$ and call $t$ the $\mathbb{R}$ coordinate. Modulo gauge transformation, we can pick a representative in temporal gauge: $A$ has no $d t$ term and can be seen as a path of connections on $T^{3}$, parameterized by $\mathbb{R}$. In temporal gauge, the Dirac operator splits as

$$
\mathfrak{D}_{A}^{*}=-\frac{\partial}{\partial t}+D_{A}
$$

with $D_{A}$ the Dirac operator on the cross-section $\{t\} \times T^{3}$. Furthermore, as $t \rightarrow \infty$ and $t \rightarrow-\infty$, the connection $A$ has flat limits $\Gamma_{+}$and $\Gamma_{-} ;$see $[21$, Theorem 4.3.1]. Consequently, the operator $D_{A_{z}}$ limits to $D_{\Gamma_{+} z}$ and $D_{\Gamma_{-} z}$ at $+\infty$ and $-\infty$. It is a crucial observation of Atiyah-Patodi-Singer [2] that the unbounded operator $\mathfrak{P}_{A_{z}}^{*}: L^{2} \rightarrow L^{2}$ is Fredholm if and only if 0 is not in the spectrum of either $D_{\Gamma_{+} z}$ or $D_{\Gamma_{-} z}$; see [6, Chapter 6] for a very detailed account.

As it turns out, any flat $\mathrm{SU}(2)$ bundle over a 3 -manifold splits as a sum of flat $U(1)$-bundles. Our bundle $E$, restricted to $\pm \infty$, splits respectively as

$$
E=L_{w_{ \pm}} \oplus L_{-w_{ \pm}}
$$

for some $w_{ \pm} \in \mathbb{R}^{3^{*}}$. The spectrum of $D_{\Gamma_{+} z}$ is thus the multiset

$$
\operatorname{Spec}\left(D_{\Gamma_{+} z}\right)= \pm 2 \pi\left|\Lambda_{\mathbb{Z}}^{*}-w_{+}-z\right| \cup \pm 2 \pi\left|\Lambda_{\mathbb{Z}}^{*}+w_{+}-z\right|
$$


for the part $\Lambda_{\mathbb{Z}}^{*} \cong \mathbb{Z}^{3}$ of $\Lambda^{*}$ in $\mathbb{R}^{3^{*}}$, and similarly for $D_{\Gamma_{-} z}$; see [6, Chapter 3].

Thus, $\mathfrak{P}_{A_{z}}^{*}$ is Fredholm as long as $z$ is not in the set

$$
W=\left\{w_{+},-w_{+}, w_{-},-w_{-}\right\}
$$

Keeping a parallel with the notation for the ADHMN story, the set $W$ is in some sense our set of "infinity data," although in a much milder way than for $\mathbb{R}^{4}$.

It is appropriate at this point to ask for which $z$ is $\mathfrak{P}_{A z}^{*}$ Fredholm when we change the domain to allow for more or less growth. Choosing a weight $\delta \in \mathbb{R}^{2}$, say $\delta=\left(\delta_{-}, \delta_{+}\right)$, and a weighing function $\sigma_{\delta}$ such that

$$
\sigma_{\delta}= \begin{cases}e^{-\delta_{-} t}, & \text { for } t<-1 \\ e^{-\delta_{+} t}, & \text { for } t>1\end{cases}
$$

we define the weighted $L^{2}$-norm

$$
\|f\|_{L_{\delta}^{2}}:=\left\|\sigma_{\delta} f\right\|_{L^{2}}
$$

and naturally

$$
L_{\delta}^{2}:=\left\{f \in L_{l o c}^{2} \mid\|f\|_{L_{\delta}^{2}}<\infty\right\} .
$$

We omit the bundle from the notation, as it should always be clear which bundle is involved.

Similarly, we can define weighted Sobolev spaces. These include only those $L_{\delta}^{2}$ sections whose derivatives are also in $L_{\delta}^{2}$. Fix a connection $\nabla$ on $E$, and set

$$
W_{\delta}^{1,2}:=\left\{f \in L_{\delta}^{2} \mid \nabla f \in L_{\delta}^{2}\right\} .
$$

Keeping in mind that the first coordinate of the weight describes the growth at $-\infty$ while the second describes the growth at $+\infty$, we define the grid

$$
\mathfrak{G}_{A}:=\operatorname{Spec}\left(D_{\Gamma_{-}}\right) \times \mathbb{R} \cup \mathbb{R} \times \operatorname{Spec}\left(D_{\Gamma_{+}}\right)
$$

in the weight space $\mathbb{R}^{2}$. Naturally, the Atiyah-Patodi-Singer condition becomes

$$
\mathfrak{P}_{A_{z}}^{*}: W_{\delta}^{1,2} \rightarrow L_{\delta}^{2} \text { is Fredholm if and only if } \delta \notin \mathfrak{G}_{A_{z}} .
$$

We define the spaces

$$
\begin{aligned}
\operatorname{ker}(\delta) & :=\operatorname{ker}\left(\mathfrak{D}_{A}: W_{\delta}^{1,2} \rightarrow L_{\delta}^{2}\right), \\
\operatorname{ker}^{*}(\delta) & :=\operatorname{ker}\left(\not{D}_{A}^{*}: W_{\delta}^{1,2} \rightarrow L_{\delta}^{2}\right),
\end{aligned}
$$


and the integers

$$
\begin{aligned}
\operatorname{ind}(\delta) & :=\operatorname{ind}\left(\mathscr{D}_{A}: W_{\delta}^{1,2} \rightarrow L_{\delta}^{2}\right) \\
N(\delta) & :=\operatorname{dim} \operatorname{ker}(\delta), \text { and } \\
N^{*}(\delta) & :=\operatorname{dim}_{\operatorname{ker}^{*}(\delta)}
\end{aligned}
$$

Since $\left(L_{\delta}^{2}\right)^{*}=L_{-\delta}^{2}$, elliptic regularity tells us that $\operatorname{dim} \operatorname{coker}\left(\mathscr{D}_{A}\right)=$ $N^{*}(-\delta)$, hence

$$
\operatorname{ind}(\delta)=N(\delta)-N^{*}(-\delta) .
$$

That the formal adjoint $\mathscr{D}_{A}^{*}$ on $W_{-\delta}^{1,2}$ is really the adjoint of $\mathfrak{D}_{A}$ on $W_{\delta}^{1,2}$ is guaranteed by the following lemma.

Lemma 4.1. The subspace $\operatorname{ker}^{*}(-\delta)$ of $L_{-\delta}^{2}=\left(L_{\delta}^{2}\right)^{*}$ kills $\operatorname{Im}(\delta)$ in the $L^{2}$ natural pairing.

Proof. Suppose $\phi$ is a smooth function with compact support. Then for all $\psi \in \operatorname{ker}^{*}(-\delta)$, we have $\langle\psi, \not{D} \phi\rangle=\left\langle\mathfrak{D}^{*} \psi, \phi\right\rangle=0$. Since $C_{c}^{\infty}$ is dense in $W_{\delta}^{1,2}$, the lemma holds.

The operator $\mathfrak{D}_{A_{z}}^{*}: W_{\delta}^{1,2} \rightarrow L_{\delta}^{2}$ is conjugate to the operator $\mathfrak{D}_{A_{z}}^{*}+$ $\sigma_{\delta} c l\left(\operatorname{grad} \sigma_{\delta}^{-1}\right)$ from $W^{1,2}$ to $L^{2}$. So the family parameterized by $\delta$ in an open square delimited by $\mathfrak{G}_{A_{z}}$ is continuous and hence has constant index. In fact, the dimensions of the kernel and the cokernel are also constant in an open square. The proof is easy and can be found in [6, Theorem 6.3-2].

As we cross a wall in $\mathfrak{G}_{A}$ to change from one open square to another, the index ind of $\mathfrak{D}_{A}$ and the index ind* of $\mathfrak{D}_{A}^{*}$ change as follows:

$$
\begin{aligned}
\operatorname{ind}(\delta) & =\operatorname{ind}(\eta)+\operatorname{dim}\left\{D_{\Gamma_{+}} \phi=-\lambda \phi\right\}, \text { and } \\
\operatorname{ind}^{*}(\delta) & =\operatorname{ind}^{*}(\eta)+\operatorname{dim}\left\{D_{\Gamma_{+}} \phi=\lambda \phi\right\}
\end{aligned}
$$

when $\delta_{+}<\eta_{+}$, and $\delta$ and $\eta$ are in adjacent open squares separated by the wall $\mathbb{R} \times\{\lambda\} \subset \mathfrak{G}_{A}$;

$$
\begin{aligned}
\operatorname{ind}(\delta) & =\operatorname{ind}(\eta)+\operatorname{dim}\left\{D_{\Gamma_{-}} \phi=-\lambda \phi\right\}, \text { and } \\
\operatorname{ind}^{*}(\delta) & =\operatorname{ind}^{*}(\eta)+\operatorname{dim}\left\{D_{\Gamma_{-}} \phi=\lambda \phi\right\}
\end{aligned}
$$

when $\delta_{-}>\eta_{-}$, and $\delta$ and $\eta$ are in adjacent open squares separated by the wall $\{\lambda\} \times \mathbb{R} \subset \mathfrak{G}_{A}$.

When the limit $\Gamma_{+}$is such that the kernel of $D_{\Gamma_{+}}$is $\{0\}$, not only are $\mathfrak{D}_{A}$ and $\mathfrak{D}_{A}^{*}$ Fredholm, as we saw above, we also have that $A$ decays exponentially 
to $\Gamma_{+}$. So there exist $\beta>0$ such that $\left|A-\Gamma_{+}\right| \leq C e^{-\beta t}$ for $t>0$; this is a consequence of [21, Theorem 5.2.2] and of the embedding of $W^{1,2}$ in bounded $C^{0}$ functions, [12, Theorem 3.4]. In that case, we have the following result on harmonic spinors.

Theorem 4.2. Suppose $\phi \in \operatorname{ker}\left(\mathfrak{D}_{A}^{*}\right) \cap W_{\delta}^{1,2}$. Suppose $\lambda-\beta<\eta<\delta$ and that $\lambda$ is the only eigenvalue of $D_{\Gamma_{+}}$between $\eta$ and $\delta: \operatorname{Spec}\left(D_{\Gamma_{+}}\right) \cap[\eta, \delta]=$ $\{\lambda\}$. Then, there exists an eigenvector $\bar{\psi}$ of $D_{\Gamma_{+}}$of eigenvalue $\lambda$ on $T^{3}$ and $\bar{\phi} \in W_{\eta}^{1,2}\left((0, \infty) \times T^{3}\right)$ such that

$$
\phi=e^{\lambda t} \bar{\psi}+\bar{\phi} \text { for } t>0
$$

Furthermore, $\bar{\phi}=O\left(e^{\eta t}\right)$ as $t \rightarrow \infty$.

Proof. The space $L^{2}\left(T^{3}\right)$ splits according to the finite dimensional eigenspaces $W_{\lambda}$ for $D_{\Gamma_{+}}$. Let $\Pi_{\delta}^{+}, \Pi_{\delta}^{-}$and $\Pi_{\delta}$ be respectively the projections from $L^{2}\left(T^{3}\right)$ to

$$
\bigoplus_{\lambda>\delta} W_{\lambda}, \bigoplus_{\lambda<\delta} W_{\lambda}, \text { and } W_{\delta}
$$

To simplify the notation, we omit $\delta$ when it is 0 and set $\phi^{ \pm}:=\Pi^{ \pm}(\phi)$.

For every $\phi \in L^{2}(Y)$, let $\phi_{\lambda}$ be its $W_{\lambda}$ component. Thus $\phi=\sum \phi_{\lambda}$. Using this decomposition, we can define the space $W^{\frac{1}{2}, 2}\left(T^{3}\right)$ using the norm

$$
\|\phi\|_{W^{\frac{1}{2}, 2}}^{2}=\sum(1+|\lambda|)\left\|\phi_{\lambda}\right\|_{L^{2}}^{2}
$$

Because $T^{3}$ is compact, the space $W^{\frac{1}{2}, 2}\left(T^{3}\right)$ defined by two different Dirac operators are equal, with commensurate norms. The + and - part of $L^{2}$, however, depend highly on $D_{\Gamma_{+}}$.

The operator

$$
\begin{aligned}
\mathfrak{D}_{\Gamma_{+}}: W^{1,2}\left([a, \infty) \times T^{3}\right) & \rightarrow L^{2}\left([a, \infty) \times T^{3}\right) \oplus \Pi^{+} W^{\frac{1}{2}, 2}\left(\{a\} \times T^{3}\right) \\
\phi & \mapsto\left(\mathscr{D}_{\Gamma_{+}} \phi, \Pi^{+} \phi(a)\right)
\end{aligned}
$$

is an isomorphism when $D_{\Gamma_{+}}$has no kernel.

The proof of this fact starts as one does in the full cylinder case:

$$
\begin{aligned}
\left\|\not_{\Gamma_{+}} \phi\right\|_{L^{2}}^{2} & =\left\|\partial_{t} \phi\right\|_{L^{2}}^{2}+\left\|D_{\Gamma_{+}} \phi\right\|_{L^{2}}^{2}+\int_{a}^{\infty} \partial_{t}\left\langle\phi, D_{\Gamma_{+}} \phi\right\rangle_{L^{2}(Y)} \\
& \geq C\|\phi\|_{W^{1,2}}^{2}-\left\langle\phi(a), D_{\Gamma_{+}} \phi(a)\right\rangle_{L^{2}(Y)}
\end{aligned}
$$


Contrary to the full cylinder case, the boundary term here cannot be made to vanish and henceforth helps control the $W^{1,2}$-norm of $\phi$. Using the inequality above and the decomposition $\phi=\sum \phi_{\lambda}$, we find

$$
\|\phi\|_{W^{1,2}}^{2} \leq C\left(\left\|\not_{\Gamma_{+}} \phi\right\|_{L^{2}}^{2}+\left\|\phi^{+}(a)\right\|_{W^{\frac{1}{2}, 2}\left(T^{3}\right)}^{2}\right) .
$$

We just proved that $\|\phi\|_{W^{1,2}} \leq C\left\|\mathfrak{D}_{\Gamma_{+}}^{\prime} \phi\right\|$, hence $\mathfrak{D}_{\Gamma_{+}}^{\prime}$ is semi-Fredholm and injective. Suppose now that $(\psi, \eta)$ is perpendicular to $\operatorname{Im}\left(\mathfrak{D}_{\Gamma_{+}}\right)$. For all $\phi \in W^{1,2}\left([a, \infty) \times T^{3}\right)$, we have

$$
\begin{aligned}
0 & =\left\langle\mathscr{D}_{\Gamma_{+}} \phi, \psi\right\rangle+\left\langle\eta, \phi^{+}(a)\right\rangle \\
& =\left\langle\phi, \mathfrak{D}_{\Gamma_{+}}^{*} \psi\right\rangle-\langle\phi(a), \psi(a)\rangle+\left\langle\eta, \phi^{+}(a)\right\rangle \\
& =\left\langle\phi, \mathscr{D}_{\Gamma_{+}}^{*} \psi\right\rangle-\left\langle\phi^{-}(a), \psi^{-}(a)\right\rangle+\left\langle\eta-\psi^{+}(a), \phi^{+}(a)\right\rangle .
\end{aligned}
$$

Testing against all the $\phi$ with $\phi(a)=0$ in a first time, $\phi^{+}(a)=0$ then, and finally $\phi^{-}(a)=0$, we prove

$$
\begin{aligned}
& \not{P}_{\Gamma_{+}}^{*} \psi=0, \\
& \eta=\psi^{+}(a), \\
& \psi^{-}(a)=0 .
\end{aligned}
$$

Thus we have $-\partial_{t} \psi+D_{\Gamma_{+}} \psi=0$, which means that $\psi$ is a linear combination of the $e^{\lambda t} \psi_{\lambda}$. The condition $\psi^{-}(a)=0$ forces out all the negative $\lambda$, while the positive ones are forced out by the $L^{2}$ condition. Hence, $\psi=0$ and $\mathfrak{D}^{!} \Gamma_{+}$ is surjective. The proof that the operator in Eq. (4.7) is an isomorphism is now complete.

For $a$ big enough, the operator $\mathfrak{D}_{A}$, not independant of $t$ but close enough to $\mathfrak{D}_{\Gamma_{+}}$, is also an isomorphism.

As in the full cylinder case, we can look at weighted version of $\not{D}$ and $\mathfrak{D} !$ For computing the asymptotic expansion of harmonic spinors, we actually need to consider the dual $\mathfrak{D}^{*}$ and its counterpart

$$
\begin{aligned}
\mathfrak{D}_{A}^{*}: W_{\delta}^{1,2}\left([a, \infty) \times T^{3}\right) & \rightarrow L_{\delta}^{2}\left([a, \infty) \times T^{3}\right) \oplus \Pi_{\delta}^{-} W^{\frac{1}{2}, 2}\left(T^{3}\right) \\
\phi & \mapsto\left(\mathfrak{P}_{A}^{*} \phi, \Pi^{-} \phi(a)\right),
\end{aligned}
$$

which is Fredholm if and only if $\delta \notin \operatorname{Spec}(D)$, and is an isomorphism when Fredholm.

We close the proof of Theorem 4.2 with a diagram chase. We first introduce maps to compose our diagram. 
Recall $\eta<\delta$ and $\operatorname{Spec}(D) \cap[\eta, \delta]=\{\lambda\}$. Then obviously, the map

$$
\begin{aligned}
I: \Pi_{\eta}^{-} W^{\frac{1}{2}, 2}\left(\{a\} \times T^{3}\right) \oplus W_{\lambda} & \rightarrow \Pi_{\delta}^{-} W^{\frac{1}{2}, 2}\left(\{a\} \times T^{3}\right) \\
(\phi, \psi) & \mapsto \phi+e^{a \lambda} \psi
\end{aligned}
$$

is an isomorphism, while the map

$$
\begin{aligned}
J: W_{\eta}^{1,2}\left([a, \infty) \times T^{3}\right) \oplus W_{\lambda} & \rightarrow W_{\delta}^{1,2}\left([a, \infty) \times T^{3}\right) \\
(\phi, \psi) & \mapsto \phi+e^{\lambda t} \psi
\end{aligned}
$$

is an injection.

Consider now the map

$K: W_{\eta}^{1,2}\left([a, \infty) \times T^{3}\right) \oplus W_{\lambda} \rightarrow L_{\eta}^{2}\left([a, \infty) \times T^{3}\right) \oplus \Pi_{\eta}^{-} W^{\frac{1}{2}, 2}\left(\{a\} \times T^{3}\right) \oplus W_{\lambda}$

$$
(\phi, \psi) \mapsto\left(\mathscr{P}_{A}\left(\phi+e^{\lambda t} \psi\right), \Pi_{\eta}^{-} \phi, \psi+e^{-a \lambda} \Pi_{\lambda} \phi(a)\right) .
$$

As $\left|\mathfrak{D}_{A}^{*}\left(e^{\lambda t} \psi\right)\right| \leq C e^{(\lambda-\beta) t}|\psi|$, then $\mathfrak{D}_{A}^{*}\left(e^{\lambda t} \psi\right) \in L_{\eta}^{2}\left([a, \infty) \times T^{3}\right)$, and $K$ is well-defined.

We put all these maps in a commutative diagram

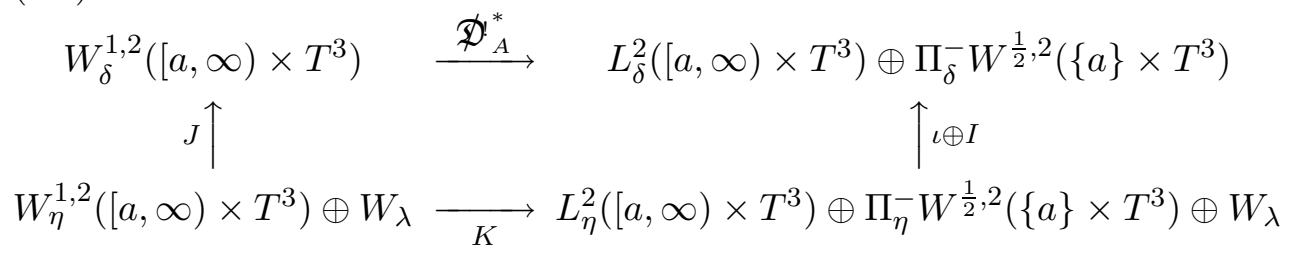

We know that $\mathfrak{D}_{A}^{*}$ is an isomorphism. Using the identification

$$
\mathfrak{D}_{A}^{*}: W_{\eta}^{1,2}\left([a, \infty) \times T^{3}\right) \equiv L_{\eta}^{2}\left([a, \infty) \times T^{3}\right) \oplus \Pi_{\eta}^{-} W^{\frac{1}{2}, 2}\left(\{a\} \times T^{3}\right),
$$

we see that $K$ has the form

$$
\left[\begin{array}{ll}
1 & p \\
q & 1
\end{array}\right]
$$

for the splitting $W_{\eta}^{1,2}\left([a, \infty) \times T^{3}\right) \oplus W_{\lambda}$ of the domain and codomain. Hence, $K-1$ is a compact operator, and $K$ is thus Fredholm of index 0. If $K(x)=$ $K(y)$, then $\mathfrak{D}_{A}^{\prime *} J(x)=\mathfrak{D}_{A}^{*} J(y)$ as the diagram is commutative, hence $x=y$ and $K$ is injective. Being of index 0 , it henceforth must be an isomorphism.

Let us now exploit this fantastic diagram. Suppose

$$
\phi \in \operatorname{ker}\left(\mathfrak{P}_{A}^{*}\right) \cap W_{\delta}^{1,2}\left(\mathbb{R} \times T^{3}\right) .
$$


Then for $a$ big enough, the diagram (4.9) has rows which are isomorphism for $\delta$ and $\eta$ satisfying the hypothesis of the theorem.

We now chase around the diagram. Since $I$ is an isomorphism, we know there exist $(\chi, \nu) \in \Pi_{\eta}^{-} W^{\frac{1}{2}, 2}\left(\{a\} \times T^{3}\right) \oplus W_{\lambda}$ such that

$$
\iota \oplus I(0, \chi, \nu)=\not \mathfrak{D}_{A}^{*}(\phi) .
$$

But as $K$ is an isomorphism, there is $(\bar{\phi}, \bar{\psi}) \in W_{\eta}^{1,2}\left([a, \infty) \times T^{3}\right) \oplus W_{\lambda}$ such that

$$
K(\bar{\phi}, \bar{\psi})=(0, \chi, \nu)
$$

By commutativity of the diagram, we have

$$
\mathfrak{D}_{A}^{\prime *} J(\bar{\phi}, \bar{\psi})=\mathfrak{D}_{A}^{\prime *}(\phi)
$$

but $\mathfrak{D}_{A}^{*}$ is an isomorphism hence $\phi=e^{\lambda t} \bar{\psi}+\bar{\phi}$ for $t>a$.

As the choice of $a$ is artificial, we set $a=0$. The proof is now complete.

Suppose now

$$
\lambda \in \operatorname{Spec}\left(D_{\Gamma_{-}}\right) \times \operatorname{Spec}\left(D_{\Gamma_{+}}\right),
$$

$\delta$ is in the upper left open square adjacent to $\lambda$,

$\eta$ is in the lower right open square adjacent to $\lambda$.

When $A$ decays exponentially to its limits, we have

$$
\operatorname{ker}(\lambda)=\operatorname{ker}(\eta)
$$

Indeed, suppose now $\phi \in \operatorname{ker}(\lambda)$. Then, $\phi \in \operatorname{ker}(\delta)$ hence by Theorem 4.2, we expand $\phi$ for $t>0$ as $\phi=e^{-\lambda_{+} t} \psi_{\lambda_{+}}+\bar{\phi}$, with $\bar{\phi} \in W_{\eta_{+}}^{1,2}\left([0, \infty) \times T^{3}\right)$. Since $\phi$ and $\bar{\phi}$ are both in $W_{\lambda_{+}}^{1,2}$, so is the term $e^{-\lambda_{+} t} \psi_{\lambda_{+}}$. This fact implies that $\psi_{\lambda_{+}}=0$. Using a similar proof at $-\infty$, we find $\phi \in W_{\eta}^{1,2}$. Obviously, the same is true for ker*.

\section{Nahm transform: Instantons to singular monopoles.}

Since $\mathfrak{D}_{A_{z}}^{*}$ is Fredholm $L^{2} \rightarrow L^{2}$ outside of $W$, and since $\operatorname{ker}\left(\mathscr{D}_{A_{z}}\right)=0$ as $F_{A_{z}}$ is ASD and $\mathbb{R} \times T^{3}$ has infinite volume, we have a bundle $V$ over $T^{3} \backslash W$ whose fiber at $z$ is

$$
V_{z}:=\operatorname{ker}\left(\mathfrak{D}_{A_{z}}^{*}\right) \cap L^{2}
$$


As outlined earlier, this bundle is equipped with

$$
\begin{aligned}
& \text { a connection } B \text { on } T^{3} \backslash W \\
& \text { a Higgs field } \Phi \in \Gamma\left(T^{3} \backslash W, \operatorname{End} V\right) \text {. }
\end{aligned}
$$

The main result of this present paper is the following theorem.

Theorem 5.1. Outside of a set $W$ consisting of at most four points, the family of vector spaces $V$ described above defines a vector bundle of rank

$$
\frac{1}{8 \pi^{2}} \int\left|F_{A}\right|^{2}
$$

and the couple $(B, \Phi)$ satisfies the Bogomolny equation

$$
\nabla_{B} \Phi=* F_{B}
$$

For $w \in W$ and $z$ close enough to $w$, there are maps $\Phi^{\perp}$ and $\Phi^{\lrcorner}$such that

$$
\Phi=\frac{-i}{2|z-w|} \Phi^{\perp}+\Phi^{\lrcorner}
$$

and $\Phi^{\perp}$ is the $L^{2}$-orthogonal projection on the orthogonal complement of a naturally defined subbundle $V$ of $V$.

The last part of the theorem is made clearer by the introduction of $V$ in Section 6.

Proof. The rank of $V$ is computed in Lemma 5.2 below.

The boundary term of Eq. (3.2) is

$$
\partial \text {-term }=\left.\left\langle\nu \Omega G \phi, d^{z} \psi\right\rangle_{T^{3}}\right|_{-\infty} ^{\infty}
$$

For $z \notin W$, both $G \phi$ and $d^{z} \psi$ decay exponentially by Theorem 4.2 hence

$$
\partial \text {-term }=0
$$

and the connection $P d^{z}$ on $\mathbb{R} \times\left(T^{3} \backslash W\right)$ is ASD. Thus, as explained in Section 3, the pair $(B, \Phi)$ satisfies outside of $W$ the appropriate dimensional reduction of the ASD equation, which is in this case the Bogomolny Equation

$$
\nabla_{B} \Phi=* F_{B}
$$


The last part of the theorem is the content of Section 7 and rests on the splitting of Section 6 .

As announced, we compute now the rank of $V$, and prove an $L^{2}$-index theorem for $\mathbb{R} \times T^{3}$.

Lemma 5.2. For a $S U(2)$-instanton $(E, A)$ on $\mathbb{R} \times T^{3}$, the index of the Dirac operator

$$
\not_{A}: W^{1,2}\left(\mathbb{R} \times T^{3}\right) \rightarrow L^{2}\left(\mathbb{R} \times T^{3}\right)
$$

when $A$ has non-zero limits at $\pm \infty$ is given by the formula

$$
\operatorname{ind}\left(\mathscr{D}_{A}\right)=-\frac{1}{8 \pi^{2}} \int\left|F_{A}\right|^{2}
$$

Proof. The fact that $A$ has non-zero limits guarantees that the operator $\mathscr{D}_{A}$ is Fredholm on $W^{1,2}$. Moreover, $A$ decays exponentially to its limits.

Let

$$
\left(\chi_{R}^{+}, \chi_{R}^{-}, \chi_{R}^{0}\right)
$$

be a partition of unity subordinate to the covering

$$
\left((R, \infty) \times T^{3},(-\infty,-R) \times T^{3},(-R-1, R+1) \times T^{3}\right) .
$$

Suppose $\Gamma_{ \pm}=d+\gamma_{ \pm}$, and $A=d+a$. Then $a$ tends to $\gamma_{+}$and $\gamma_{-}$when $t$ tends to $+\infty$ and $-\infty$ respectively. Set

$$
a_{R}=\chi_{R}^{+} \gamma_{+}+\chi_{R}^{-} \gamma_{-}+\chi_{R}^{0} a .
$$

The sequence $\mathscr{D}_{a_{n}}-\mathscr{D}_{a_{R}}$ of compact operators is Cauchy, and thus has a limit, $K$ say, which is then compact. As $\mathscr{D}_{A}=\mathscr{D}_{a_{R}}+K$, we have that $\operatorname{ind}\left(\mathscr{P}_{A}\right)=\operatorname{ind}\left(\mathscr{D}_{a_{R}}\right)$ for all $R>0$. We now compute $\operatorname{ind}\left(\mathscr{P}_{a_{R}}\right)$ using the relative index theorem. It could be that $\Gamma_{-} \neq \Gamma_{+}$, but this case is easily converted to a situation where $\Gamma_{-}=\Gamma_{+}$, as we now see.

Choose a path $\Gamma_{s}$ in the space of flat connections on $T^{3}$ starting at $\Gamma_{+}$ and ending at $\Gamma_{-}$, and avoiding the trivial connection. Hence, $0 \notin \operatorname{Spec}\left(D_{\Gamma_{s}}\right)$ for all $s$; recall Eq. (4.1). Suppose $\Gamma_{s}=d+\gamma_{s}$ and set

$$
a_{R}^{s}=\chi_{R}^{+} \gamma_{s}+\chi_{R}^{-} \gamma_{-}+\chi_{R}^{0} a .
$$

The family $\mathfrak{P}_{a_{R}^{s}}$ of Fredholm operator depends continuously on $s$. Hence

$$
\operatorname{ind}\left(\mathscr{D}_{A}\right)=\operatorname{ind}\left(\mathscr{D}_{a_{R}}\right)=\operatorname{ind}\left(\mathscr{D}_{a_{R}^{0}}\right)=\operatorname{ind}\left(\mathscr{D}_{a_{R}^{1}}\right) .
$$


Note that the connection $a_{R}^{1}$ equals $\Gamma_{-}$outside $[-R-1, R+1] \times T^{3}$. Hence the relative index theorem tells us

$$
\operatorname{ind}\left(\mathscr{P}_{a_{R}^{1}}\right)-\operatorname{ind}\left(\mathscr{P}_{\Gamma_{-}}\right)=\operatorname{ind}\left(\tilde{\mathscr{P}}_{a_{R}^{1}}\right)-\operatorname{ind}\left(\tilde{\mathscr{P}}_{\Gamma_{-}}\right),
$$

where the tilded operators are extensions to some compact manifold of the restriction of the operators $\mathscr{D}_{a_{R}^{1}}$ and $\mathscr{D}_{\Gamma_{-}}$to $[-R-1, R+1] \times T^{3}$.

Because $D_{\Gamma_{-}}$has no kernel, $\mathscr{D}_{\Gamma_{-}}: W^{1,2} \rightarrow L^{2}$ is an isomorphism, and thus ind $\left(\mathscr{D}_{\Gamma_{-}}\right)=0$. Hence, the left-hand side of Eq. (5.3) is equal to ind $\left(\mathscr{D}_{A}\right)$.

To compute the right-hand side, we embed $[-R-1, R+1] \times T^{3}$ in some flat $T^{4}$. The spinor bundles $S^{+}$and $S^{-}$on $[-R-1, R+1] \times T^{3}$ agree very nicely with those of $T^{4}$. We extend both $a_{R}^{1}$ and $\Gamma_{-}$by the trivial bundle with connection $\Gamma_{-}$.

The Atiyah-Singer index theorem tells us that

$$
\begin{aligned}
\operatorname{ind}\left(\tilde{\mathfrak{P}}_{\Gamma_{-}}\right) & =\left\{\operatorname{ch}\left(\Gamma_{-}\right) \cdot \hat{\mathbf{A}}\left(T^{4}\right)\right\}\left[T^{4}\right] \\
\operatorname{ind}\left(\tilde{\mathscr{P}}_{a_{R}^{1}}\right) & =\left\{\operatorname{ch}\left(a_{R}^{1}\right) \cdot \hat{\mathbf{A}}\left(T^{4}\right)\right\}\left[T^{4}\right] \\
& =\left(\frac{c_{1}^{2}}{2}-c_{2}\right)\left[T^{4}\right] .
\end{aligned}
$$

Since $a_{R}^{1}$ is in $\mathrm{SU}(2)$, we have $c_{1}=0$, while

$$
c_{2}\left[T^{4}\right]=\frac{1}{8 \pi^{2}} \int_{T^{4}}\left|\left(\left|F_{a_{R}^{1}}^{-}\right|^{2}-\left|F_{a_{R}^{1}}^{+}\right|^{2}\right)\right|^{2} .
$$

Note that on the complement of $[-R-1, R+1] \times T^{3}$ in $T^{4}$, the connection $a_{R}^{1}$ equals $\Gamma_{-}$hence is flat there. Furthermore, on $[-R, R] \times T^{3}$, we have $a_{R}^{1}=A$. On $[R, R+1] \times T^{3}$ and $[-R-1,-R] \times T^{3}$, the curvature $F_{a_{R}^{1}}$ involves cut off functions, their derivatives and $\left(A-\Gamma_{-}\right)$terms. Since $A$ tends to $\Gamma_{-}$exponentially fast, we therefore have constant $C$ and $\beta$ such that

$$
\left.\left|\operatorname{ind}\left(\oiint_{A}\right)+\frac{1}{8 \pi^{2}} \int_{[-R, R] \times T^{3}}\right| F_{A}\right|^{2} \mid \leq C e^{-\beta R} .
$$

As $R \rightarrow \infty$, we have the wanted result.

\section{A geometric splitting and exact sequences.}

In this section, we analyze a splitting of $V$ in a neighborhood of a point $w \in W$ where the solution $(B, \phi)$ to Bogomolny equation is singular. This 
point $w$ is associated, say, to the limit $\Gamma=\Gamma_{+}$of $A$ at $+\infty$, in the sense that $\Gamma$ splits $E$ as $L_{w} \oplus L_{-w}$ on $T^{3}$.

Suppose the connection $A$ decays at most with rate $\beta$, as in $\left|A-\Gamma_{+}\right| \leq$ $C e^{-\beta t}$ for $t>0$ and $\left|A-\Gamma_{-}\right| \leq C e^{\beta t}$ for $t<0$. Set

$$
\epsilon:=\frac{1}{4} \min \left(\beta, \operatorname{dist}\left(w, \Lambda^{*}+W \backslash\{w\}\right)\right),
$$

and define the six weights

$$
\begin{array}{lll}
\ulcorner\epsilon:=(-\epsilon, \epsilon) & \bar{\epsilon}:=(0, \epsilon) & \bar{\epsilon}:=(\epsilon, \epsilon) \\
\epsilon:=(-\epsilon,-\epsilon) & \underline{\epsilon}:=(0,-\epsilon) & \epsilon=:=(\epsilon,-\epsilon)
\end{array}
$$

displayed here in a way which is reminiscent of their position in $\mathbb{R}^{2}$.

Consider the ball $B^{3}(w)$ of radius $2 \epsilon$ around $w$. As $z$ varies in $B^{3}(w)$, and depending on whether $\Gamma_{+}=\Gamma_{-}$or not, there are two or one walls to cross to pass from 0 to $\ulcorner\epsilon$ and from $\epsilon$ to 0 . In a picture, we have

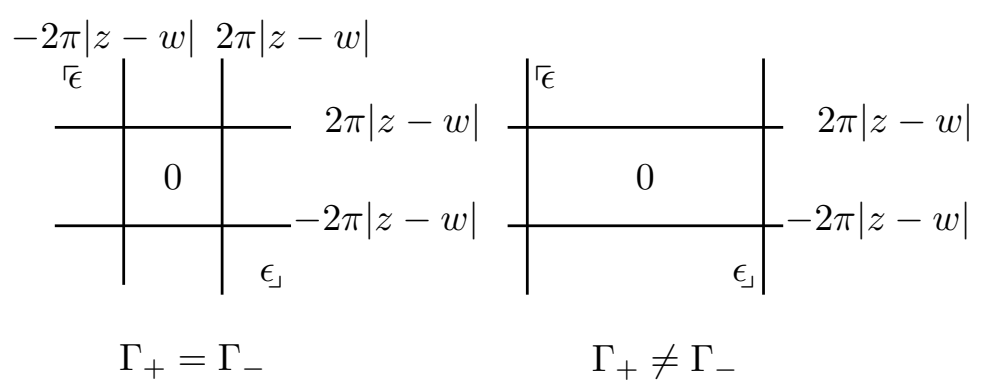

As $z$ varies in $B^{3}(w)$, those walls move around without ever touching $\epsilon_{\lrcorner}$and $\Gamma_{\epsilon}$. Hence, for $L_{\epsilon}^{2}$ and $L_{\Gamma_{\epsilon}}^{2}$, the operators $\mathscr{D}_{A_{z}}, \mathfrak{D}_{A_{z}}^{*}$ and $\mathfrak{D}_{A_{z}}^{*} \mathscr{D}_{A_{z}}$ are Fredholm for all $z \in B^{3}(w)$.

Hence, for $z \in B^{3}(w)$, the six vector spaces

$$
\begin{array}{rlrl}
V_{z} & :=\operatorname{ker}\left(\mathfrak{P}_{A_{z}}^{*}\right) \cap L_{\Gamma_{\epsilon}}^{2}, & K_{z}:=\operatorname{ker}\left(\mathscr{D}_{A_{z}}\right) \cap L_{\Gamma_{\epsilon}}^{2}, \\
V_{z} & :=\operatorname{ker}\left(\mathfrak{D}_{A_{z}}^{*}\right) \cap L_{\epsilon_{\lrcorner}}^{2}, & K_{z}:=\operatorname{ker}\left(\mathscr{D}_{A_{z}}\right) \cap L^{2} \\
\mathcal{H}_{z} & :=\operatorname{ker}\left(\nabla_{A_{z}}^{*} \nabla_{A_{z}}\right) \cap L_{\Gamma_{\epsilon}}^{2}, & & K_{\exists}:=\operatorname{ker}\left(\mathfrak{D}_{A_{z}}\right) \cap L_{\epsilon_{\lrcorner}}^{2},
\end{array}
$$

are kernels of Fredholm operators. By contrast, the space $V_{z}$, already defined as $\operatorname{ker}\left(\mathfrak{D}_{A_{z}}^{*}\right) \cap L^{2}$, is not the kernel of a Fredholm operator at $w$.

Notice that none of those vector spaces form a priori a bundle over $B^{3}(w)$ as the dimensions could jump at random. However, for $L_{\epsilon \epsilon}^{2}$ and $L_{\epsilon}^{2}$, the operators $\mathfrak{D}_{A_{z}}, \mathfrak{D}_{A_{z}}^{*}$, and $\nabla_{A_{z}}^{*} \nabla_{A_{z}}$ are Fredholm operators for all 
$z \in B^{3}(w)$. The various indices are therefore constant and we have that, for example,

$$
\operatorname{dim} V_{z}-\operatorname{dim} K_{z} \text { is constant on } B^{3}(w) .
$$

We have the following obvious results:

$$
\begin{array}{lr}
V \subset V \subset \bar{C}, & K \subset K \subset \bar{C}, \\
\not{D} \mathcal{H} \subset \overline{ } \subset^{\top}, & \bar{K} \subset \mathcal{H}, \\
& K=K=\{0\} .
\end{array}
$$

Equation (4.10) signifies here that $V_{w}=V_{w}$. The following few lemmas describe in more detail the relationship between the various spaces.

The smallest eigenvalues of $D_{\Gamma_{z}}$ are $\pm 2 \pi|z-w|$. For simplicity, we set

$$
\lambda:=2 \pi|z-w|
$$

and define

$$
W_{\lambda}:=\lambda \text { eigenspace of } D_{\Gamma_{z}} \text { on } T^{3} .
$$

The family $W_{\lambda}$ defines a bundle over the sphere $|z-w|=\lambda / 2 \pi$ around $w$. Its rank is given by

$$
\operatorname{rk} W_{\lambda}= \begin{cases}1, & \text { if } \lambda \neq 0 \text { and } 2 w \notin \Lambda^{*} ; \\ 2, & \text { if } \lambda \neq 0 \text { and } 2 w \in \Lambda^{*}, \text { or } \lambda=0 \text { and } 2 w \notin \Lambda^{*} ; \\ 4, & \text { if } \lambda=0 \text { and } 2 w \in \Lambda^{*} .\end{cases}
$$

This $W_{\lambda}$ plays an important role in understanding the relations between the various spaces just introduced.

For any instanton connection $A^{\prime}$ on $\mathbb{R} \times T^{3}$, set

$$
\begin{aligned}
V(\delta) & :=\operatorname{ker}\left(\mathfrak{D}_{A^{\prime}}^{*}\right) \cap L_{\delta}^{2}, \\
K(\delta) & :=\operatorname{ker}\left(\mathscr{D}_{A^{\prime}}\right) \cap L_{\delta}^{2},
\end{aligned}
$$

and let $[\delta]$ denote the open square in $\mathbb{R}^{2} \backslash \mathfrak{G}_{A^{\prime}}$ containing $\delta$.

Lemma 6.1 (one wall). Suppose $\delta, \eta \in \mathbb{R}^{2} \backslash \mathfrak{G}_{A^{\prime}}$ are weights for which $[\delta]$ and $[\eta]$ are adjacent and separated by the wall $\{\mu\} \times \mathbb{R}$ or $\mathbb{R} \times\{\mu\}$. Then, the sequence

$0 \longrightarrow V(\delta) \longrightarrow V(\eta) \stackrel{\lim \left(e^{-\mu t} .\right)}{\longrightarrow} W_{\mu} \stackrel{\left(\lim \left(e^{\mu t} \cdot\right)\right)^{*}}{\longrightarrow} K(-\delta)^{*} \longrightarrow K(-\eta)^{*} \longrightarrow 0$, where the limits are both evaluated at $+\infty$ when $[\eta]$ is above $[\delta]$ and at $-\infty$ when $[\eta]$ is to the left of $[\delta]$, is exact. 
Proof. Theorem 4.2 ensures that the limits give functions $\alpha$ and $\beta^{*}$ which are well defined, and that

$$
0 \longrightarrow V(\delta) \longrightarrow V(\eta) \longrightarrow W_{\mu} \quad \text { and } \quad 0 \longrightarrow K(-\eta) \longrightarrow K(-\delta) \longrightarrow W_{\mu}
$$

are exact.

It only remains to prove that Sequence (6.2) is exact at $W_{\mu}$. Suppose $\phi \in V(\eta)$ and $\psi \in K(-\delta)$. Then,

$$
\begin{aligned}
0 & =\left\langle\mathfrak{D}_{A^{\prime}}^{*} \phi, \psi\right\rangle-\left\langle\phi, \mathfrak{D}_{A^{\prime}} \psi\right\rangle \\
& =\lim _{t \rightarrow \infty}\left\langle\phi, c l\left(\frac{\partial}{\partial t}\right) \psi\right\rangle-\lim _{t \rightarrow-\infty}\left\langle\phi, c l\left(\frac{\partial}{\partial t}\right) \psi\right\rangle \\
& =\lim _{t \rightarrow \infty}\left\langle e^{-\mu t} \phi, c l\left(\frac{\partial}{\partial t}\right) e^{\mu t} \psi\right\rangle-\lim _{t \rightarrow-\infty}\left\langle e^{-\mu t} \phi, c l\left(\frac{\partial}{\partial t}\right) e^{\mu t} \psi\right\rangle .
\end{aligned}
$$

One of those limits is $\beta^{*} \alpha(\phi)(\psi)$ while the other one vanishes as we now see. Suppose $[\eta]$ is above $[\delta]$, and suppose $\left\{\mu^{\prime}\right\} \times \mathbb{R}$ is the wall to their right. Then, $\phi=O\left(e^{\mu^{\prime} t}\right)$ as $t \rightarrow-\infty$ by Theorem 4.2. But for some $\mu^{\prime \prime}<\mu^{\prime}$, the wall $\left\{-\mu^{\prime \prime}\right\} \times \mathbb{R}$ is exactly to the right of $[-\eta]$; hence, $\psi=O\left(e^{-\mu^{\prime \prime} t}\right)$ as $t \rightarrow-\infty$. But then

$$
\beta^{*} \alpha(\phi)(\psi)=\lim _{t \rightarrow-\infty} O\left(e^{\left(\mu^{\prime}-\mu^{\prime \prime}\right) t}\right)=0,
$$

hence $\operatorname{Im}(\alpha) \subset \operatorname{ker}\left(\beta^{*}\right)$. A similar argument establishes the same fact when $[\eta]$ is to the left of $[\delta]$.

The sequence is then exact if $\operatorname{dim} \operatorname{Im}(\alpha)=\operatorname{dim} \operatorname{ker}\left(\beta^{*}\right)$. We have two short exact sequences:

$$
\begin{gathered}
0 \longrightarrow V(\delta) \longrightarrow V(\eta) \longrightarrow \operatorname{Im}(\alpha) \longrightarrow 0, \quad \text { and } \\
0 \longrightarrow W_{\mu} / \operatorname{ker}\left(\beta^{*}\right) \longrightarrow K(-\delta)^{*} \longrightarrow K(-\eta)^{*} \longrightarrow 0 .
\end{gathered}
$$

Using those short exact sequences and notation from Eq. (4.3), we have

$$
\begin{aligned}
\operatorname{dim} \operatorname{Im}(\alpha)-\operatorname{dim} \operatorname{ker}\left(\beta^{*}\right) & =N^{*}(\eta)-N^{*}(\delta)-\operatorname{dim} W_{\mu}+N(-\delta)-N(-\eta) \\
& =\operatorname{ind}^{*}(\eta)-\operatorname{ind}^{*}(\delta)-\operatorname{dim} W_{\mu} .
\end{aligned}
$$

The Wall Crossing Equation (4.4) forces the last line to be 0. The proof is thus complete.

Corollary 6.2. Suppose $\Gamma_{+} \neq \Gamma_{-}$. Then the sequences

$$
\begin{aligned}
0 \longrightarrow V_{z} \longrightarrow V_{z} \longrightarrow W_{\lambda} \longrightarrow 0, & \text { for } \lambda \neq 0, \\
0 \longrightarrow V_{z} \longrightarrow V_{z} \longrightarrow W_{-\lambda} \longrightarrow K_{z} \longrightarrow 0, & \text { for } \lambda \neq 0, \\
0 \longrightarrow V_{w} \longrightarrow V_{w} \longrightarrow W_{0} \longrightarrow K_{w} \longrightarrow 0, &
\end{aligned}
$$


are exact.

Proof. Apply Lemma 6.1 to the choice of weights $\left\{\lceil\epsilon, 0\}\right.$ and $\left\{0, \epsilon_{\lrcorner}\right\}$for the connection $A^{\prime}=A_{z}$, and remember that $K=K=\{0\}$.

Corollary 6.3. Suppose $\Gamma_{+}=\Gamma_{-}$. Then the sequences

$$
\begin{aligned}
0 \longrightarrow V_{z} \longrightarrow V_{z} \longrightarrow W_{\lambda} \oplus W_{-\lambda} \longrightarrow 0, & \text { for } \lambda \neq 0 \\
0 \longrightarrow V_{z} \longrightarrow V_{z} \longrightarrow W_{\lambda} \oplus W_{-\lambda} \longrightarrow K_{z} \longrightarrow 0, & \text { for } \lambda \neq 0 \\
0 \longrightarrow V_{w} \longrightarrow V_{w} \longrightarrow W_{0} \oplus W_{0} \longrightarrow K_{w} \longrightarrow 0 &
\end{aligned}
$$

are exact.

Proof. Suppose we have the following choice of weights:

\begin{tabular}{c|c}
$\delta$ & $\delta$ \\
\hline$\delta$ & $\delta$
\end{tabular}

Denote $\iota$ any inclusion map, and $L_{\mu}^{ \pm}$the maps

$$
L_{\mu}^{+}(\phi)=\lim _{t \rightarrow \infty} e^{\mu t} \phi, \quad \text { and } \quad L_{\mu}^{-}=\lim _{t \rightarrow-\infty} e^{\mu t} \phi
$$

Then sequences akin to Sequence (6.2) fit in a diagram

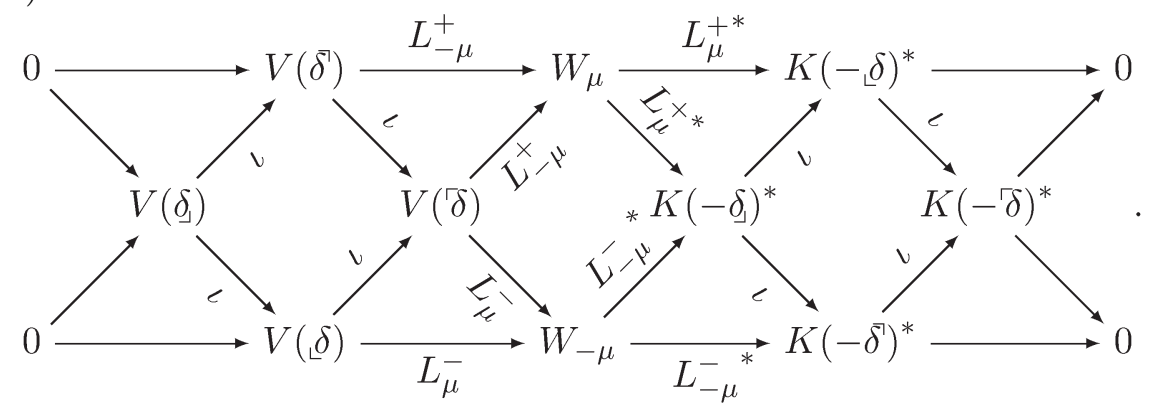


Suppose $\phi \in V(\delta)$, and $\psi \in K\left(-\delta_{\lrcorner}\right)$. Then

$$
\begin{aligned}
0 & =\left\langle\not_{A^{\prime}} \phi, \psi\right\rangle-\left\langle\phi, \mathfrak{D}_{A^{\prime}} \psi\right\rangle \\
& =\left.\left\langle\phi, c l\left(\frac{\partial}{\partial t}\right) \psi\right\rangle\right|_{-\infty} ^{\infty} \\
& =\lim _{t \rightarrow \infty}\left\langle e^{-\mu t} \phi, c l\left(\frac{\partial}{\partial t}\right) e^{\mu t} \psi\right\rangle-\lim _{t \rightarrow-\infty}\left\langle e^{\mu t} \phi, c l\left(\frac{\partial}{\partial t}\right) e^{-\mu t} \psi\right\rangle \\
& =\left(L_{\mu}^{+*} L_{-\mu}^{+}(\phi)-L_{-\mu}^{-}{ }^{*} L_{\mu}^{-}(\phi)\right)(\psi),
\end{aligned}
$$

hence the middle square commutes. It is quite obvious that all the other squares and triangles commute. From Diagram (6.9), we extract, for an obvious choice of maps, the exact sequence

$$
0 \longrightarrow V\left(\delta_{\lrcorner}\right) \longrightarrow V(\delta) \longrightarrow W_{\mu} \oplus W_{-\mu} \longrightarrow K(-\delta)^{*} \longrightarrow K(-\delta)^{*} \longrightarrow 0 .
$$

In particular, the sets of weights
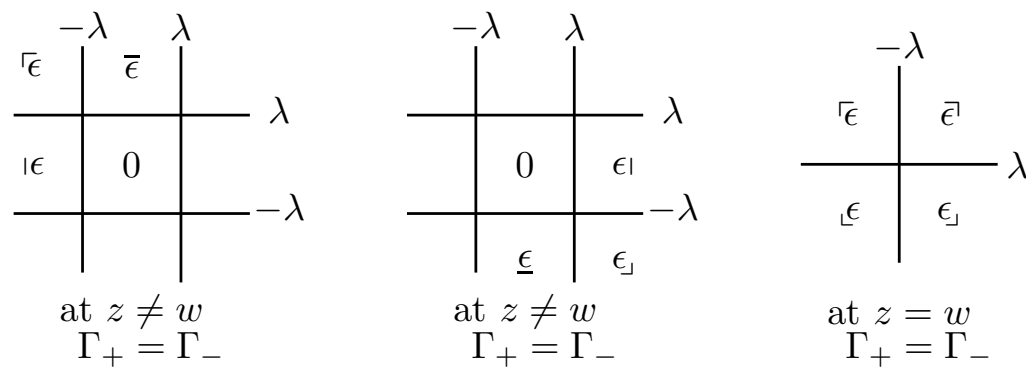

yield for $A^{\prime}=A_{z}$ the exact sequences (6.6), (6.7) and (6.8).

An analysis for $\nabla_{A_{z}}^{*} \nabla_{A_{z}}$ brings a very similar wall crossing formula

$$
\operatorname{ind}\left(\nabla_{A_{z}}^{*} \nabla_{A_{z}}, \Gamma_{\epsilon}\right)-\operatorname{ind}\left(\nabla_{A_{z}}^{*} \nabla_{A_{z}}, \epsilon_{\lrcorner}\right)= \begin{cases}2 \operatorname{dim} W_{0}, & \text { for } \Gamma_{+} \neq \Gamma_{-} ; \\ 4 \operatorname{dim} W_{0}, & \text { for } \Gamma_{+}=\Gamma_{-} .\end{cases}
$$

However, since $\nabla_{A_{z}}^{*} \nabla_{A_{z}}$ is self-adjoint, ind $\left(\nabla_{A_{z}}^{*} \nabla_{A_{z}}, \Gamma_{\epsilon}\right)=-\operatorname{ind}\left(\nabla_{A_{z}}^{*} \nabla_{A_{z}}, \epsilon_{\lrcorner}\right)$, whence

$$
\operatorname{rk} \mathcal{H}= \begin{cases}\operatorname{dim} W_{0}, & \text { for } \Gamma_{+} \neq \Gamma_{-} \\ 2 \operatorname{dim} W_{0}, & \text { for } \Gamma_{+}=\Gamma_{-} .\end{cases}
$$

Using Equation (6.1), we can even say

$$
\operatorname{rk\mathcal {H}}= \begin{cases}2, & \text { for } \Gamma_{+} \neq \Gamma_{-} \text {and } 2 w \notin \Lambda^{*} ; \\ 4, & \text { for } \Gamma_{+} \neq \Gamma_{-} \text {and } 2 w \in \Lambda^{*}, \text { or } \Gamma_{+}=\Gamma_{-} \text {and } 2 w \notin \Lambda^{*} \\ 8, & \text { for } \Gamma_{+}=\Gamma_{-} \text {and } 2 w \in \Lambda^{*} .\end{cases}
$$


Similarly, we have for the Laplacian the following isomorphisms:

$0 \longrightarrow \mathcal{H}_{z} \longrightarrow\left(W_{\lambda} \oplus W_{-\lambda}\right)^{\oplus 2} \longrightarrow 0, \quad$ for $z \neq w$ and when $\Gamma_{+}=\Gamma_{-}$, (6.13) $0 \longrightarrow \mathcal{H}_{w} \longrightarrow W_{0} \oplus W_{0} \longrightarrow 0, \quad$ when $\Gamma_{+}=\Gamma_{-}$.

Bringing all of those sequences together allows us to conclude the following.

Theorem 6.4. On $B^{3}(w)$, we have

$$
{ }^{\top}=V \oplus \not{D H}
$$

Proof. Denote $W_{\lambda}^{\prime}$ the space

$$
W_{\lambda}^{\prime}:= \begin{cases}W_{\lambda} \oplus W_{-\lambda}, & \text { if } \Gamma_{+}=\Gamma_{-} \\ W_{\lambda}, & \text { if } \Gamma_{+} \neq \Gamma_{-} .\end{cases}
$$

Let $p: W_{\lambda}^{\prime} \oplus W^{\prime}{ }_{-\lambda} \rightarrow W^{\prime}{ }_{\lambda}$ denote the map $p(a, b)=2 \lambda a$.

For $\lambda \neq 0$, we use the Snake Lemma on the diagram

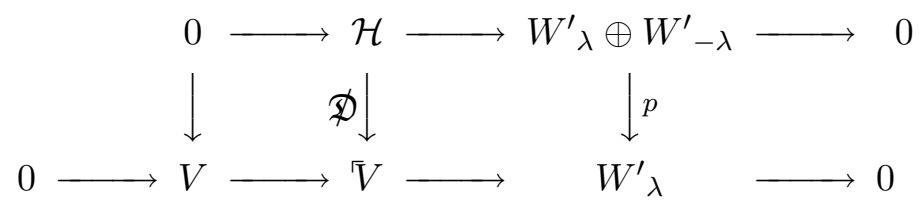

coming from Sequences (6.3), (6.6), (6.10), and (6.12), to produce an exact sequence

$$
\begin{gathered}
\operatorname{ker}(0) \longrightarrow \operatorname{ker}(\not)) \\
\quad \longrightarrow \operatorname{ker}(p) \longrightarrow \operatorname{coker}(0) \longrightarrow \operatorname{coker}(\not)) \longrightarrow \operatorname{coker}(p) \\
\quad \longrightarrow K_{z} \longrightarrow W^{\prime} \longrightarrow V_{z} \longrightarrow \operatorname{coker}(\not{D}) \longrightarrow 0
\end{gathered}
$$

Note that the map $V \rightarrow \operatorname{coker}(\not{D})$ being surjective forces $V$ to be spanned by $V$ and $\mathfrak{D H}$.

Sequences (6.4) and (6.7) imply

$$
\operatorname{dim} V_{z}=\operatorname{dim} V_{\lrcorner}+\operatorname{dim} W_{\lambda}^{\prime}-\operatorname{dim} K_{z}
$$


while Sequences (6.3) and (6.6) imply

$$
\operatorname{dim} V_{z}=\operatorname{dim} V_{z}+\operatorname{dim} W_{\lambda}^{\prime} .
$$

Thus,

$$
\operatorname{dim} V_{z}=\operatorname{dim} V_{z}+2 \operatorname{dim} W_{\lambda}^{\prime}-\operatorname{dim} K_{z}=\operatorname{dim} V_{z}+\operatorname{dim} \not{D} \mathcal{H} .
$$

Since Lemma 4.1 guarantees that $\langle\not{D} \mathcal{H}, V\rangle=\{0\}$, we have $V \cap \mathfrak{D} \mathcal{H}$ perpendicular to $V$ for the $L^{2}$ inner product. Hence, $\mathscr{D H} \cap V=\{0\}$, and $\bar{V}_{z}=V_{z} \oplus \not{D} \mathcal{H}$.

It remains to prove the theorem for $z=w$. We already know $V_{w}=V_{w}$ and $\mathfrak{D H}_{w} \subset{ }^{\sqrt{ }} V_{w}$. We also know from Sequences (6.5) and (6.8) that

$$
\begin{aligned}
\operatorname{dim} V_{w} & =\operatorname{dim} V_{w}+\operatorname{dim} W^{\prime}{ }_{0}-\operatorname{dim} K_{w} \\
& =\operatorname{dim} V_{w}+\operatorname{dim} \not{D} \mathcal{H}_{w} .
\end{aligned}
$$

We therefore only have to prove that the intersection $V_{w} \cap \mathfrak{D}_{A_{w}} \mathcal{H}_{w}$ is $\{0\}$ to complete the proof.

The asymptotic behavior of $\phi \in \mathcal{H}_{w}$ is

$$
\phi= \begin{cases}t \phi_{0}^{+}+\phi_{1}^{+}+o(1), & \text { as } t \rightarrow \infty ; \\ t \phi_{0}^{-}+\phi_{1}^{-}+o(1), & \text { as } t \rightarrow-\infty ;\end{cases}
$$

for some $\phi_{0}^{ \pm}, \phi_{1}^{ \pm} \in W_{0}$. If $\Gamma_{+} \neq \Gamma_{-}$, we must have $\phi_{0}^{-}=\phi_{1}^{-}=0$, as $w$ is associated to $\Gamma_{+}$.

The asymptotic behavior of $\mathscr{D}_{A_{w}} \phi$ is

$$
\mathscr{D}_{A_{w}} \phi= \begin{cases}\phi_{0}^{+}+o(1), & \text { as } t \rightarrow \infty \\ \phi_{0}^{-}+o(1), & \text { as } t \rightarrow-\infty\end{cases}
$$

Suppose $\mathfrak{D}_{A_{w}} \phi \in L^{2}$. Then,

$$
\begin{aligned}
\left\|\mathfrak{P}_{A_{w}} \phi\right\|_{L^{2}}^{2}= & \left\langle\mathfrak{D}_{A_{w}}^{*} \mathfrak{D}_{A_{w}} \phi, \phi\right\rangle+\lim _{t \rightarrow \infty}\left\langle\mathfrak{D}_{A_{w}} \phi, c l\left(\frac{\partial}{\partial t}\right) \phi\right\rangle \\
& +\lim _{t \rightarrow-\infty}\left\langle\mathfrak{P}_{A_{w}} \phi, c l\left(\frac{\partial}{\partial t}\right) \phi\right\rangle \\
= & \left\langle\phi_{0}^{+}, \phi_{1}^{+}\right\rangle+\lim _{t \rightarrow \infty} t\left|\phi_{0}^{+}\right|^{2}-\left\langle\phi_{0}^{-}, \phi_{1}^{-}\right\rangle-\lim _{t \rightarrow-\infty} t\left|\phi_{0}^{-}\right|^{2} .
\end{aligned}
$$

For $\left\|\mathscr{D}_{A_{w}} \phi\right\|_{L^{2}}$ to be finite, we must get rid of the limits, thus forcing $\phi_{0}^{ \pm}=0$ and consequently, we have $\mathfrak{D}_{A_{w}} \phi=0$. The proof is now complete. 
For a continuous family of Fredholm operators, like $\mathscr{D}_{A_{z}}$ on $L_{\bar{\epsilon}}^{2}$ parameterized on $B^{3}(w)$, the dimension of the kernel can only drop in a small neighborhood of a given point, it cannot increase. However, not any random behavior is acceptable.

Lemma 6.5 (see [20, p. 241]). Let $T: X \rightarrow Y$ be Fredholm and $S: X \rightarrow$ $Y$ a bounded operator. Then the operator $T+t S$ is Fredholm and $\operatorname{dim} \operatorname{ker}(T+$ $t S)$ is constant for small $|t|>0$.

We obviously use this lemma with $T=\mathscr{D}_{A_{w}}, X=W_{\Gamma_{\epsilon}}^{1,2}, Y=L_{\digamma_{\epsilon}}^{2}$, and $S=\operatorname{cl}(e)$ for some direction $e \in \mathbb{R}^{3}$. Let us note that three scenarios are possible.

1. $\operatorname{dim} K_{z}$ is constant on a neighborhood around $w$, say $B^{3}(w)$;

2. $\operatorname{dim} K_{z}$ is constant for $z \in B^{3}(w) \backslash\{w\}$, but is smaller than $\operatorname{dim} K_{w}$;

3. $\operatorname{dim} K_{w+\lambda e} \neq \operatorname{dim} K_{w+\lambda^{\prime} e^{\prime}}$ for small $\lambda, \lambda^{\prime}>0$ and some $e \neq e^{\prime}$.

\section{Asymptotic of the Higgs field.}

We now study the behavior of the Higgs field $\Phi$ as $z$ approaches of a given element $w$ of $W$. We know $w$ is associated to the limit $\Gamma$ of $A$ at $\infty$ or $-\infty$, in the sense that $\Gamma$ splits $E$ as $L_{w} \oplus L_{-w}$. Without loss of generality, we suppose

$$
\Gamma_{+}=\Gamma
$$

When $\Gamma_{+} \neq \Gamma_{-}$, and for $2 \pi|z-w|<\epsilon$, notice that

$$
\begin{aligned}
V_{z} & =L_{\leftarrow \epsilon}^{2} \cap \operatorname{ker}\left(\mathfrak{P}_{A_{z}}^{*}\right)=L_{\bar{\epsilon}}^{2} \cap \operatorname{ker}\left(\mathfrak{P}_{A_{z}}^{*}\right)=L_{\bar{\epsilon}}^{2} \cap \operatorname{ker}\left(\mathfrak{P}_{A_{z}}^{*}\right), \text { and } \\
V_{z} & =L_{\epsilon \epsilon}^{2} \cap \operatorname{ker}\left(\mathfrak{P}_{A_{z}}^{*}\right)=L_{\underline{\epsilon}}^{2} \cap \operatorname{ker}\left(\mathfrak{P}_{A_{z}^{*}}\right)=L_{\epsilon}^{2} \cap \operatorname{ker}\left(\mathfrak{P}_{A_{z}}^{*}\right) .
\end{aligned}
$$

When $\Gamma_{+}=\Gamma_{-}$, those spaces are a priori all different.

Theorem 7.1. On a closed ball $B^{3}(w)$ around $w$, there exist families of operators $\Phi^{\perp}$ and $\Phi^{\lrcorner}$, bounded independently of $z$, such that

$$
\Phi=\frac{-i}{2|z-w|} \Phi^{\perp}+\Phi^{\lrcorner}
$$

Furthermore, $\Phi^{\perp}$ is the $L^{2}$-orthogonal projection on $\mathfrak{D}_{A_{z}} \mathcal{H}_{z} \cap V_{z}$. 
Proof. Obviously, $V$ supports many different norms, and amongst those are the $L^{2}$ and $L_{\epsilon}^{2}$ norms. For $\phi \in V_{\lrcorner}$, observe that

$$
\|t \phi\|_{L^{2}} \leq C_{\epsilon}\|\phi\|_{L_{\epsilon}^{2}}
$$

We would really like to bound this last quantity by a multiple of $\|\phi\|_{L^{2}}$.

Let $Q$ denote the projection $L^{2} \rightarrow V_{w}$. Of course, since $L_{\epsilon}^{2} \subset L^{2}$, the projection is also defined on $L_{\epsilon}^{2}$. Let $V_{w}^{\perp}$ be the $L^{2}$-orthogonal complement, and $V_{w}^{0}=V_{w}^{\perp} \cap L_{\epsilon}^{2}$. In fact, we have

$$
L_{\epsilon}^{2}=V_{w} \oplus V_{w}^{0}
$$

since at $w$, we have $V_{w}=V_{w}$.

Since $\mathscr{D}_{A_{w}}^{*}$ is injective on $V_{w}^{0}$, there is a constant such that

$$
\|u\|_{L_{\epsilon^{2}}^{2}} \leq C\left\|\mathfrak{D}_{A_{w}}^{*} u\right\|_{L_{\epsilon^{2}}^{2}} \text { for } u \in V_{w}^{0} .
$$

But then for $u \in V_{w+\lambda e}$, we have

$$
\begin{aligned}
& \|u\|_{L_{\epsilon^{\prime}}^{2}} \leq\|Q u\|_{L_{\epsilon^{2}}^{2}}+\|(1-Q) u\|_{L_{\epsilon^{\prime}}^{2}} \\
& \leq\|Q u\|_{L_{\epsilon_{\lrcorner}}^{2}}+C\left\|\mathfrak{P}_{A_{w}}^{*}(1-Q) u\right\|_{L_{\epsilon_{\lrcorner}}^{2}} \\
& =\|Q u\|_{L_{\epsilon_{\lrcorner}}^{2}}+C\left\|\mathfrak{P}_{A_{w}}^{*} u\right\|_{L_{\epsilon^{\prime}}^{2}} \\
& =\|Q u\|_{L_{\epsilon^{\prime}}^{2}}+C \lambda\|u\|_{L_{\epsilon^{2}}^{2}} \text {. }
\end{aligned}
$$

Hence, for $\lambda$ small enough,

$$
\|u\|_{L_{\epsilon}^{2}} \leq 2\|Q u\|_{L_{\epsilon^{2}}^{2}}
$$

Of course, since $V_{w}$ is finite dimensional, there exists a constant $C$ for which $\|Q u\|_{L_{\epsilon}^{2}} \leq C\|Q u\|_{L^{2}}$ and thus for $u \in V_{\lrcorner z}$ with $z$ close to $w$,

$$
\|t u\|_{L^{2}} \leq C_{\epsilon}\|u\|_{L_{\epsilon^{2}}^{2}} \leq 2 C_{\epsilon}\|Q u\|_{L_{\epsilon^{2}}^{2}} \leq C\|Q u\|_{L^{2}} \leq C\|u\|_{L^{2}}
$$

Denote $P^{\lrcorner}$the $L^{2}$-orthogonal projection of $V$ on $V$. We just proved that

$$
\Phi \circ P^{\lrcorner} \text {is bounded independently of } z \in B^{3}(w) \text {. }
$$

It is part of the map $\Phi^{\lrcorner}$announced in the statement of the theorem.

One of the crucial features of this proof is our ability to find a uniform bound for $m_{t}$ on $V$. 
As suggested above, let $\Phi^{\perp}$ denote the $L^{2}$-orthogonal projection on $\mathscr{D}_{A_{z}} \mathcal{H}_{z} \cap V_{z}$. Then,

$$
\begin{aligned}
\Phi=-2 \pi i P m_{t} & =\Phi P^{\lrcorner}-2 \pi i\left(P^{\lrcorner}+\Phi^{\perp}\right) m_{t} \Phi^{\perp} \\
& =\Phi P^{\lrcorner}+2 \pi i P^{\lrcorner} m_{t} \Phi^{\perp}-2 \pi i \Phi^{\perp} m_{t} \Phi^{\perp} .
\end{aligned}
$$

For $\phi_{1} \in V$, and $\phi_{2} \in V$, we have $\left\langle\phi_{1}, t \Phi^{\perp} \phi_{2}\right\rangle=\left\langle t \phi_{1}, \Phi^{\perp} \phi_{2}\right\rangle$. Thus, $P^{\lrcorner} m_{t} \Phi^{\perp}$ is also bounded independently of $z \in B^{3}(w)$. Let

It remains only to analyze $\Phi^{\perp} m_{t} \Phi^{\perp}$. Pick a vector $e \in \mathbb{R}^{3}$ of length 1 .

$$
\mathcal{R}=\left\{w+\frac{\lambda}{2 \pi} e\right\} \subset B^{3}(w)
$$

be a ray inside $B^{3}(w)$ emerging from $w$. As the notation suggests, we parameterize this ray by $\lambda=2 \pi|z-w|$. Pick a family $\phi_{z} \in \mathscr{D}_{A_{z}} \mathcal{H}_{z}$ for $z \in \mathcal{R}$, with

$$
\begin{gathered}
\phi_{z} \in V_{z} \text { for } \lambda>0, \\
\left\|\phi_{z}\right\|_{L_{L_{\epsilon}}^{2}}=1 .
\end{gathered}
$$

But then,

$$
\left\|\phi_{z}\right\|_{L^{2}} \rightarrow \infty \text { as } \lambda \rightarrow 0
$$

To prove this claim, suppose it is not true. Then, there is a subsequence $\phi_{z_{j}} \rightarrow \tilde{\phi}_{w}$ weakly in $L^{2}$. Hence, $\left\langle\phi_{z_{j}}, f\right\rangle \rightarrow\left\langle\tilde{\phi}_{w}, f\right\rangle$ for all $f \in L^{2}$, in particular for all $f \in L_{\epsilon}^{2}=\left(L_{\tau_{\epsilon}}^{2}\right)^{*}$, whence $\phi_{z_{j}} \rightarrow \tilde{\phi}_{w}$ weakly in $L_{\tau_{\epsilon}}^{2}$. Since $\phi_{z} \rightarrow \phi_{w}$ in $L_{\tau_{\epsilon}}^{2}$, we have $\tilde{\phi}_{w}=\phi_{w}$, which is impossible as $\tilde{\phi}_{w}$ is in $L^{2}$ while $\phi_{w}$ is not.

Because $\Gamma_{w}$ is independent of $t$, and because $-\epsilon$ is not an eigenvalue of $D_{\Gamma_{w}}$, the operator $\mathfrak{P}_{\Gamma_{w}}^{*}$ is an isomorphism $W_{\llcorner\epsilon}^{1,2} \rightarrow L_{\llcorner\epsilon}^{2}$, and $W_{\epsilon}^{1,2} \rightarrow L_{\epsilon}^{2}$, hence there exists a constant $C$ such that

$$
\begin{array}{ll}
\|u\|_{W_{\llcorner\epsilon}^{1,2}} \leq C\left\|\mathfrak{P}_{\Gamma_{w}}^{*} u\right\|_{L_{\llcorner\epsilon}^{2}}, & \text { for } u \in W_{\llcorner}^{1,2}, \\
\|u\|_{W_{\epsilon}^{1,2}} \leq C\left\|\mathscr{P}_{\Gamma_{w}}^{*} u\right\|_{L_{\epsilon}^{2}}, & \text { for } u \in W_{\epsilon}^{1,2} .
\end{array}
$$

Because $\phi_{z} \in V_{z}$ for $\lambda>0$, for $t>0$, we can write $\phi_{z}=$ $e^{-\lambda t} \psi_{-\lambda}+g_{z}$ for some eigenvector $\psi_{-\lambda}$ of eigenvalue $-\lambda$ of $D_{\Gamma_{z}}$ and some $g_{z} \in W_{-\epsilon}^{1,2}\left([0, \infty) \times T^{3}\right)$. When $\Gamma_{-}=\Gamma_{+}$, and for $t<0$, we can write $\phi_{z}=e^{\lambda t} \psi_{\lambda}+j_{z}$ for some eigenvector $\psi_{\lambda}$ of eigenvalue $\lambda$ of $D_{\Gamma_{z}}$ and some $j_{z} \in W_{\epsilon}^{1,2}\left((-\infty, 0] \times T^{3}\right)$. 
While $g_{z}$ and $j_{z}$ appear to be defined only for $t>0$ and $t<0$ respectively, let us define them globally on $\mathbb{R} \times T^{3}$ by $g_{z}=\phi_{z}-e^{-\lambda t} \psi_{-\lambda}$ and $j_{z}=$ $\phi_{z}-e^{\lambda t} \psi_{\lambda}$.

Notice that

$$
\mathfrak{P}_{\Gamma_{z}}^{*} g_{z}=\mathfrak{D}_{\Gamma_{z}}^{*} \phi_{z}=\left(\mathfrak{P}_{\Gamma_{z}}^{*}-\mathfrak{D}_{A_{z}}^{*}\right) \phi_{z}=\operatorname{cl}(\Gamma-A) \phi_{z},
$$

and similarly

$$
\mathfrak{P}_{\Gamma_{z}}^{*} j_{z}=\mathfrak{P}_{\Gamma_{z}}^{*} \phi_{z}=\left(\mathfrak{P}_{\Gamma_{z}}^{*}-\mathscr{D}_{A_{z}}^{*}\right) \phi_{z}=\operatorname{cl}(\Gamma-A) \phi_{z},
$$

Overall, there is a constant such that $|c l(A-\Gamma)| \leq C \sigma_{(0, \beta)}$, and this estimate can be improved to $|c l(A-\Gamma)| \leq C \sigma_{(-\beta, \beta)}$ when $\Gamma_{-}=\Gamma_{+}$. Hence, $\operatorname{cl}(A-\Gamma)$ gives a bounded map $L_{\Gamma \epsilon}^{2} \rightarrow L_{\llcorner\epsilon}^{2}$ in all cases and $L_{\leftarrow_{\epsilon}}^{2} \rightarrow L_{\epsilon}^{2}$ when $\Gamma_{-}=\Gamma_{+}$. Thus, Eq. (7.5) yields

$$
\left\|\mathfrak{P}_{\Gamma_{z}}^{*} g_{z}\right\|_{L_{\llcorner\epsilon}^{2}} \leq C\left\|\phi_{z}\right\|_{L_{\Gamma \epsilon}^{2}}
$$

and for the special case $\Gamma_{-}=\Gamma_{+}$, Eq. (7.6) yields

$$
\left\|\not{P}_{\Gamma_{z}}^{*} j_{z}\right\|_{L_{\epsilon}^{2}} \leq C\left\|\phi_{z}\right\|_{L_{\Gamma_{\epsilon}}^{2}}
$$

From Eqs. (7.3), and (7.7), we derive

$$
\begin{aligned}
\left\|g_{z}\right\|_{W_{\llcorner\epsilon}^{1,2}} & \leq C\left\|\mathfrak{P}_{\Gamma_{w}}^{*} g_{z}\right\|_{L_{\llcorner\epsilon}^{2}} \\
& =C\left\|\mathfrak{P}_{\Gamma_{z}}^{*} g_{z}+\lambda c l(e) g_{z}\right\|_{L_{\llcorner\epsilon}^{2}} \\
& \leq C\left\|\phi_{z}\right\|_{L_{\leftarrow \epsilon}^{2}}+C \lambda\left\|g_{z}\right\|_{L_{\llcorner\epsilon}^{2}},
\end{aligned}
$$

After rearranging, we notice that $\left\|g_{z}\right\|_{W_{\llcorner\epsilon}^{1,2}}$ is bounded independently of small $z$, and similarly $\left\|j_{z}\right\|_{W_{\bar{\epsilon}}^{1,2}}$ is bounded independently of small z. This last fact is also true for $\Gamma_{-} \neq \Gamma_{+}$, for in that case $j_{z}=\phi_{z}$ and its $L_{\Gamma_{\epsilon}}^{2}$-norm is equivalent to the $L_{\epsilon}^{2}$-norm, as both as defined on $V$ over $B^{3}(w)$.

While it is agreeable to work with a smooth splitting, nothing prevents us from considering the functions

$$
h_{\lambda}=\left\{\begin{array}{ll}
e^{\lambda t} \psi_{\lambda}, & \text { for } t<0, \\
e^{-\lambda t} \psi_{-\lambda}, & \text { for } t>0,
\end{array} \quad \text { and } \quad r_{z}= \begin{cases}j_{z}, & \text { for } t<0, \\
g_{z}, & \text { for } t>0,\end{cases}\right.
$$

and the associate splitting

$$
\phi_{z}=h_{\lambda}+r_{z}
$$


That $\left\|r_{z}\right\|_{L_{\epsilon}^{2}}$ is bounded independently of small $z$ follows from the similar fact concerning $g_{z}$ and $j_{z}$.

Consider the families

$$
\begin{aligned}
\bar{\phi}_{z} & :=\phi_{z} /\left\|\phi_{z}\right\|_{L^{2}}, \\
\bar{h}_{\lambda} & :=h_{\lambda} /\left\|\phi_{z}\right\|_{L^{2}}, \\
\bar{r}_{z} & :=r_{z} /\left\|\phi_{z}\right\|_{L^{2}} .
\end{aligned}
$$

Since $\left\|\phi_{z}\right\|_{L^{2}} \rightarrow \infty$ and $\left\|r_{z}\right\|_{L_{\epsilon^{2}}^{2}}$ is bounded, we have $\left\|\bar{r}_{z}\right\|_{L_{\epsilon^{2}}^{2}} \rightarrow 0$ as $\lambda \rightarrow 0$, and a fortiori, $\left\|\bar{r}_{z}\right\|_{L^{2}} \rightarrow 0$. The triangle inequality then guarantees

$$
\left|\left\|\bar{h}_{\lambda}\right\|_{L^{2}}-\left\|\bar{r}_{z}\right\|_{L^{2}}\right| \leq\left\|\bar{\phi}_{z}\right\|_{L^{2}} \leq\left\|\bar{h}_{\lambda}\right\|_{L^{2}}+\left\|\bar{r}_{z}\right\|_{L^{2}} .
$$

Since $\left\|\bar{\phi}_{z}\right\|_{L^{2}}=1$, and $\left\|\bar{r}_{z}\right\|_{L^{2}} \rightarrow 0$, we must have

$$
\left\|\bar{h}_{\lambda}\right\|_{L^{2}} \rightarrow 1 \text { as } \lambda \rightarrow 0
$$

Let us now come back to our main worry. We study

$$
\left\langle t \bar{\phi}_{z}, \bar{\phi}_{z}\right\rangle=\left\langle t \bar{h}_{\lambda}, \bar{h}_{\lambda}\right\rangle+2\left\langle\bar{h}_{\lambda}, t \bar{r}_{z}\right\rangle+\left\langle t \bar{r}_{z}, \bar{r}_{z}\right\rangle .
$$

The last two terms are bounded by a multiple of $\left\|t \bar{r}_{z}\right\|_{L^{2}}$. But

$$
\left\|t \bar{r}_{z}\right\|_{L^{2}} \leq C\left\|\bar{r}_{z}\right\|_{L_{\epsilon}^{2}}
$$

hence it is going to 0 .

As for the first term, we have

$$
\begin{aligned}
\left\langle t \bar{h}_{\lambda}, \bar{h}_{\lambda}\right\rangle & =\frac{1}{\left\|\phi_{\lambda}\right\|_{L^{2}}^{2}}\left(\int_{0}^{\infty} t e^{-2 \lambda t}\left|\psi_{-\lambda}\right|^{2}+\int_{-\infty}^{0} t e^{2 \lambda t}\left|\psi_{\lambda}\right|^{2}\right) \\
& =\frac{1}{2 \lambda} \frac{1}{\left\|\phi_{\lambda}\right\|_{L^{2}}^{2}}\left(\int_{0}^{\infty} e^{-2 \lambda t}\left|\psi_{-\lambda}\right|^{2}+\int_{-\infty}^{0} e^{2 \lambda t}\left|\psi_{\lambda}\right|^{2}\right) \\
& =\frac{1}{2 \lambda}\left\|\bar{h}_{\lambda}\right\|_{L^{2}}^{2},
\end{aligned}
$$

hence

$$
\left\langle t \bar{\phi}_{\lambda}, \bar{\phi}_{\lambda}\right\rangle=\frac{1}{2 \lambda}+o(1) \text { as } \lambda \rightarrow 0 .
$$

Suppose now $\bar{\phi}_{z}^{1}$ and $\bar{\phi}_{z}^{2}$ are two such families, but so that

$$
\left\langle\bar{\phi}_{z}^{1}, \bar{\phi}_{z}^{2}\right\rangle_{L^{2}}=0
$$


Then

$$
\begin{aligned}
\left\langle t \bar{\phi}_{z}^{1}, \bar{\phi}_{z}^{2}\right\rangle & =\left\langle t \bar{h}_{\lambda}^{1}, \bar{h}_{\lambda}^{2}\right\rangle+\left\langle\bar{h}_{\lambda}^{1}, t \bar{r}_{z}^{2}\right\rangle+\left\langle t \bar{r}_{z}^{1}, \bar{h}_{\lambda}^{2}\right\rangle+\left\langle t \bar{r}_{z}^{1}, \bar{r}_{z}^{2}\right\rangle \\
& =\frac{1}{2 \lambda}\left\langle\bar{h}_{\lambda}^{1}, \bar{h}_{\lambda}^{2}\right\rangle+o(1)
\end{aligned}
$$

and of course $\left\langle\bar{h}_{\lambda}^{1}, \bar{h}_{\lambda}^{2}\right\rangle \rightarrow 0$, hence the result.

Finally, let us note that in fact, Scenario 3 of page 206 cannot happen. We can take the trace of $(B, \Phi)$ to obtain an abelian monopole $(b, \varphi)$ on $B^{3}(w) \backslash\{w\}$. The Bogomolny equation reduces to

$$
d \varphi=* d b
$$

and thus $\Delta \varphi=0$. Since $\varphi$ is harmonic, not every possible behavior is acceptable as $z \rightarrow w$. For one thing, there is a unique set of homogeneous harmonic polynomials $p_{m}$ and $q_{m}$ of degree $m$ which give a decomposition of $\varphi$ on $B^{3}(w) \backslash\{w\}$ as a Laurent series

$$
\varphi=\sum_{m=0}^{\infty} p_{m}(z-w)+\sum_{m=0}^{\infty} \frac{q_{m}(z-w)}{|z-w|^{2 m+1}}
$$

see for example [3, Theorem 10.1, p. 209].

Whether or not the rank is constant, we can find for any sequence of points approaching $w$ a subsequence of points $z_{j} \rightarrow w$ for which the decomposition of Eq. 7.1 is valid. We then have

$$
\lim _{j \rightarrow \infty} 2\left|z_{j}-w\right| \varphi_{z_{j}}=i \operatorname{dim} \not{D}_{A_{z_{j}}} \mathcal{H}_{z_{j}}=i\left(\operatorname{rk} \mathcal{H}-\operatorname{dim} K_{z_{j}}\right)
$$

By the Laurent series decomposition given above, this number must be the same in any way we approach $w$, hence $\operatorname{dim} K_{z}$ must be constant on $B^{3}(w) \backslash$ $\{w\}$.

\section{Acknowledgments.}

This work is part of my Ph.D. thesis [6]. Grateful for the guidance and support of my advisor Tomasz Mrowka, I thank him warmly. I also thank Larry Guth, Peter Kronheimer, Frédéric Rochon, and Michael Singer for stimulating discussions. 


\section{References.}

[1] Michael F. Atiyah, Vladimir G. Drinfel'd, Nigel J. Hitchin, and Yuri I. Manin, Construction of instantons, Phys. Lett. A 65(3), (1978), 185187.

[2] Michael F. Atiyah, Vijay K. Patodi, and Isadore M. Singer, Spectral asymmetry and Riemannian geometry. I, Math. Proc. Cambridge Philos. Soc. 77, (1975), 43-69.

[3] Sheldon Axler, Paul Bourdon, and Wade Ramey, Harmonic function theory, second ed., Graduate Texts in Mathematics, vol. 137, SpringerVerlag, New York, 2001.

[4] Olivier Biquard and Marcos Jardim, Asymptotic behaviour and the moduli space of doubly-periodic instantons, J. Eur. Math. Soc. (JEMS) 3(4), (2001), 335-375, arXiv:math.DG/0005154.

[5] Peter J. Braam and Pierre van Baal, Nahm's transformation for instantons, Comm. Math. Phys. 122(2), (1989), 267-280.

[6] Benoit Charbonneau, Analytic aspects of periodic instantons, Ph.D. thesis, Massachusetts Institute of Technology, Cambridge, MA, USA, September 2004, can be obtained on http://theses.mit.edu or by emailing benoit@alum.mit.edu.

[7] Sergey Cherkis and Anton Kapustin, Nahm transform for periodic monopoles and $\mathcal{N}=2$ super Yang-Mills theory, Comm. Math. Phys. 218(2), (2001), 333-371, arXiv:hep-th/0006050.

[8] Sergey Cherkis and Anton Kapustin, Singular monopoles and supersymmetric gauge theories in three dimensions, Nuclear Phys. B 525(1-2), (1998), 215-234, arXiv:hep-th/9711145.

[9] Sergey Cherkis and Anton Kapustin, Singular Monopoles and Gravitational Instantons, Comm. Math. Phys. 203, (1999), 713-728, arXiv:hep-th/9803160.

[10] E. Corrigan and P. Goddard, Construction of instanton and monopole solutions and reciprocity, Ann. Physics 154(1), (1984), 253-279.

[11] Simon K. Donaldson and Peter B. Kronheimer, The geometry of fourmanifolds, Oxford Mathematical Monographs, The Clarendon Press Oxford University Press, New York, 1990. 
[12] Emmanuel Hebey, Nonlinear analysis on manifolds: Sobolev spaces and inequalities, volume 5 of Courant Lecture Notes in Mathematics, New York University Courant Institute of Mathematical Sciences, New York, 1999.

[13] Nigel J. Hitchin, On the construction of monopoles, Comm. Math. Phys. 89(2), (1983), 145-190.

[14] Jacques Hurtubise and Michael K. Murray, On the construction of monopoles for the classical groups, Comm. Math. Phys. 122(1), (1989), $35-89$.

[15] Marcos Jardim, Construction of doubly-periodic instantons, Comm. Math. Phys. 216(1), (2001), 1-15, arXiv:math.DG/9909069.

[16] Marcos Jardim, Classification and existence of doubly-periodic instantons, Q. J. Math. 53(4), (2002), 431-442, arXiv:math.DG/0108004.

[17] Marcos Jardim, Nahm transform and spectral curves for doublyperiodic instantons, Comm. Math. Phys. 225(3), (2002), 639-668, arXiv:math.DG/9910120.

[18] Marcos Jardim, A survey on Nahm transform, Journal of Geometry and Physics 52(3), (2004), 313-327, arXiv:math.DG/0309305.

[19] Marcos Jardim, Nahm transform of doubly-periodic instantons, Ph.D. thesis, Oxford, 1999, arXiv:math.DG/9912028.

[20] Tosio Kato, Perturbation theory for linear operators, Classics in Mathematics, Springer-Verlag, Berlin, 1995.

[21] John W. Morgan, Tomasz Mrowka, and Daniel Ruberman, The $L^{2}$ moduli space and a vanishing theorem for Donaldson polynomial invariants, Monographs in Geometry and Topology, II, International Press, Cambridge, MA, 1994.

[22] Werner Nahm, Self-dual monopoles and calorons, Group theoretical methods in physics (Trieste, 1983), Lecture Notes in Phys., vol. 201, Springer, Berlin, 1984, pp. 189-200.

[23] Hiraku Nakajima, Monopoles and Nahm's equations, Einstein metrics and Yang-Mills connections (Sanda, 1990), Lecture Notes in Pure and Appl. Math., vol. 145, Dekker, New York, 1993, pp. 193-211. 
[24] Tom M. W. Nye, The geometry of calorons, Ph.D. thesis, University of Edinburgh, 2001, arXiv:hep-th/0311215.

[25] Tom M. W. Nye and Michael A. Singer, An $L^{2}$-index theorem for Dirac operators on $S^{1} \times \mathbf{R}^{3}$, J. Funct. Anal. 177(1), (2000), 203-218, arXiv:math.DG/0009144.

[26] Marc Pauly, Monopole moduli spaces for compact 3-manifolds, Math. Ann. 311(1), (1998), 125-146.

[27] H. Schenk, On a generalised Fourier transform of instantons over flat tori, Comm. Math. Phys. 116(2), (1988), 177-183.

[28] Pierre van Baal, Instanton moduli for $T^{3} \times \mathbb{R}$, Nuclear Phys. B Proc. Suppl. 49, (1996), 238-249, arXiv:hep-th/9512223.

[29] Pierre van Baal, Nahm gauge fields for the torus, Phys. Letters B448, (1999), 26-32, arXiv:hep-th/9811112.

Department of Mathematics

MCGILL UNIVERSITY

805 Sherbrooke West

MontréAL, QC H3A 2K6

CANADA

E-mail address: benoit@alum.mit.edu

Received OCtober 20, 2004. 